\title{
OH populations and temperatures from simultaneous spectroscopic observations of 25 bands
}

\author{
S. Noll ${ }^{1}$, W. Kausch ${ }^{2,1}$, S. Kimeswenger ${ }^{3,1}$, S. Unterguggenberger ${ }^{1}$, and A. M. Jones ${ }^{1,4}$ \\ ${ }^{1}$ Institute for Astro- and Particle Physics, University of Innsbruck, Technikerstr. 25/8, 6020 Innsbruck, Austria \\ ${ }^{2}$ Department of Astrophysics, University of Vienna, Türkenschanzstr. 17, 1180 Vienna, Austria \\ ${ }^{3}$ Instituto de Astronomía, Universidad Católica del Norte, Avenida Angamos 0610, Antofagasta, Chile \\ ${ }^{4}$ Max Planck Institute for Astrophysics, Karl-Schwarzschild-Str. 1, 85748 Garching, Germany \\ Correspondence to: S. Noll (stefan.noll@uibk.ac.at)
}

Received: 12 November 2014 - Published in Atmos. Chem. Phys. Discuss.: 23 December 2014

Revised: 10 March 2015 - Accepted: 13 March 2015 - Published: 1 April 2015

\begin{abstract}
OH}$ rotational temperatures are widely used to derive mesopause temperatures and their variations. Since most data sets are only based on a fixed set of lines of a single band, it is important to know possible systematic uncertainties related to the choice of lines. Therefore, a comprehensive study of as many $\mathrm{OH}$ bands as possible is desirable. For this purpose, astronomical echelle spectrographs at large telescopes are the most suitable instruments. They offer a wide wavelength coverage, relatively high spectral resolution, and high sensitivity. Moreover, since each groundbased astronomical observation has an imprint of the Earth's atmosphere, the data archives of large astronomical facilities are a treasure for atmospheric studies. For our project, we used archival data of the medium-resolution X-shooter echelle spectrograph operated by the European Southern Observatory at Cerro Paranal in Chile. The instrument can simultaneously observe all $\mathrm{OH}$ bands that are accessible from ground. We reduced and analysed a set of 343 high-quality spectra taken between 2009 and 2013 to measure $\mathrm{OH}$ line intensities and to derive rotational and vibrational temperatures of 25 bands between 0.58 and $2.24 \mu \mathrm{m}$. We studied the influence of the selected line set, $\mathrm{OH}$ band, upper vibrational level $v^{\prime}$, and the molecular data on the derived level populations and temperatures. The rotational temperature results indicate differences by several degrees depending on the selection. The temperatures for bands of even and odd $v^{\prime}$ show deviations which increase with $v^{\prime}$. A study of the temporal variations revealed that the nocturnal variability pattern changes for $v^{\prime}$ from 2 to 9 . In particular, the spread of temperatures tends to increase during the night, and the time of the min-
\end{abstract}

imum temperature depends on $v^{\prime}$. The vibrational temperatures depend on the range of $v^{\prime}$ used for their determination, since the higher vibrational levels from 7 to 9 seem to be overpopulated compared to the lower levels. The vibrational temperature tends to increase during the night, while the intensity decreases. Our results support the assumption that the $\mathrm{OH}$ emission altitude depends on $v^{\prime}$. Moreover, the emission layer appears to rise in the course of the night, which makes the $\mathrm{OH}$ thermalisation less efficient. The derived rotational temperatures and their change with $v^{\prime}$ seem to be significantly affected by non-equilibrium populations.

\section{Introduction}

Analysing temperature variations in the Earth's mesopause region between 80 and $100 \mathrm{~km}$ is important for studying the chemistry, dynamics, and climate evolution in the upper atmosphere. As in situ measurements can only be performed by rockets, the most popular approach is based on groundor satellite-based observations of chemiluminescent airglow emissions (e.g. Beig et al., 2003, 2008; Khomich et al., 2008). In particular, the hydroxyl $(\mathrm{OH})$ airglow (Meinel, 1950a), which consists of strong roto-vibrational bands over a wide wavelength range from the visual to the infrared (Osterbrock et al., 1996; Rousselot et al., 2000), is suitable. The emission peak is typically at about $87 \mathrm{~km}$ with a full width at half maximum (FWHM) of about $8 \mathrm{~km}$ (e.g. Baker and Stair, 1988). Excited OH up to the vibrational level $v=9$ is 
essentially produced by the reaction

$\mathrm{H}+\mathrm{O}_{3} \rightarrow \mathrm{OH}(v \leq 9)+\mathrm{O}_{2}$

(Bates and Nicolet, 1950). The contribution of another reaction involving hydroperoxyl $\left(\mathrm{HO}_{2}\right)$ and atomic oxygen with a vibrational excitation up to level $v=6$ appears to be negligible (Xu et al., 2012). Temperatures can be estimated by assuming a Boltzmann distribution for the population of the upper states of the $\mathrm{OH}$ lines. Considering lines of different upper vibrational levels $v^{\prime}$ leads to vibrational pseudo temperatures of about $10000 \mathrm{~K}$ (e.g. Khomich et al., 2008), since the higher $v$ can only be excited by chemical reactions. However, the energy differences of the lowest rotational levels of a single $\mathrm{OH}$ band seem to be small enough that the line intensity distribution is ruled by the ambient temperature, which is of the order of about $200 \mathrm{~K}$. Consequently, $\mathrm{OH}$ rotational temperatures $T_{\text {rot }}$ are usually only derived from the first three or four rotational levels (Perminov and Semenov, 1992; Beig et al., 2003; Cosby and Slanger, 2007).

Many specialised instruments for $\mathrm{OH} T_{\text {rot }}$ determinations focus on a single $\mathrm{OH}$ band (Beig et al., 2003, 2008; Schmidt et al., 2013). (3-1), (6-2), and (8-3) are the most frequently used $\mathrm{OH}\left(v^{\prime}-v^{\prime \prime}\right)$ bands. For a comparison of the world-wide temperature measurements and an evaluation of their reliability, it is important to understand possible systematic differences related to the choice of band and line set. Satellite observations and modelling results indicate emission peaks depending on $v^{\prime}$, which also vary with time (Adler-Golden, 1997; Xu et al., 2012; von Savigny et al., 2012; Kowalewski et al., 2014). This implies band-specific $\mathrm{OH} T_{\text {rot }}$ due to the mesopause temperature profile and possible variations in the degree of thermalisation, particularly for high emission altitudes. There, the frequency of the essential collisions with the atmospheric main constituents for the relaxation process could become critical. For a better understanding of these processes, studies of the line intensities of many $\mathrm{OH}$ bands with different $v^{\prime}$ and their change with time are required. A comprehensive study should also involve an evaluation of the effect of the uncertainties still present in the $\mathrm{OH}$ line parameters, i.e. Einstein coefficients and level energies (Mies, 1974; Goldman et al., 1998; van der Loo and Groenenboom, 2007; Khomich et al., 2008).

Observations of multiple $\mathrm{OH}$ bands below $1.1 \mu \mathrm{m}$ were carried out with different medium-resolution spectrographs covering several $100 \mathrm{~nm}$ at Zvenigorod $\left(55.7^{\circ} \mathrm{N}\right)$ in Russia since the 1960s (Krassovsky et al., 1977; Perminov et al., 2007; Khomich et al., 2008). These studies resulted in $T_{\text {rot }}$ that typically showed an increase with $v^{\prime}$. Apart from an incomplete local thermodynamic equilibrium (LTE) for the higher $v^{\prime}$, real temperature differences were assumed due to $v^{\prime}$-dependent altitude differences. The variations of $T_{\text {rot }}$ of adjacent $v^{\prime}$ also indicated the best correlations. Takahashi and Batista (1981) observed the $\mathrm{OH}$ bands (5-1), (6-2), (7$2)$, (8-3), and (9-4) with a tilting filter photometer at $\mathrm{Ca}-$ choeira Paulista in Brazil $\left(22.7^{\circ} \mathrm{S}\right)$. Their data of 64 nights also showed variations depending on $v^{\prime}$. In terms of the number of analysed lines and bands, Cosby and Slanger (2007) published so far the most detailed study. It is based on $16 \mathrm{OH}$ bands in the range up to $1.06 \mu \mathrm{m}$. Their sample of $81 \mathrm{spec}-$ tra was taken with the medium-resolution Echellette Spectrograph and Imager (ESI, Sheinis et al., 2002) at Mauna Kea (Hawaii, $19.8^{\circ} \mathrm{N}$ ) in March and October 2000. Cosby and Slanger (2007) confirmed for $v^{\prime}$ from 3 to 9 , a dependence of $T_{\text {rot }}$ on $v^{\prime}$. They found minima for $v^{\prime}=3$ and maxima for $v^{\prime}=8$. Moreover, data of one night indicated a correlation of $T_{\text {rot }}$ differences for $v^{\prime}=9$ and 3 with vibrational level population ratios derived from the same $v^{\prime}$. They explained this behaviour by an altitude-dependent (and hence $v^{\prime}$-dependent) thermal equilibration efficiency by collisions, which affects the vibrational as well as the rotational relaxation. Consequently, $v^{\prime}$-related $T_{\text {rot }}$ differences have an important nonLTE component. Cosby and Slanger (2007) also concluded that the $\mathrm{OH}$ line parameters of Goldman et al. (1998) were the most reliable of those available at the time of the publication.

Although the reported studies had an important influence on our current knowledge of the physical and chemical processes in the upper atmosphere, they were still limited in the number of simultaneously observed bands, data quality, spectroscopic resolution, and/or sample size. Therefore, it is important to test the interpretations with a data set of higher quality. Cosby and Slanger (2007) used data from an echelle spectrograph. These instruments detect multiple high diffraction orders simultaneously in different wavelength ranges, which are separated by another perpendicularly mounted dispersive element. By merging the different order spectra, a wide wavelength range can be covered at a relatively high resolution, which is well suited for simultaneous observations of several $\mathrm{OH}$ bands. Echelle spectrographs are frequently used in astronomy. Since each ground-based astronomical observation also has an imprint of the Earth's atmosphere, these data can be used to study the atmospheric $\mathrm{OH}$ bands. In fact, the ESI data of Cosby and Slanger (2007) were plain night-sky observations at the astronomical Keck II telescope.

The European Southern Observatory (ESO) operates the Very Large Telescope (VLT) at the Cerro Paranal in Chile $\left(2635 \mathrm{~m}, 24^{\circ} 38^{\prime} \mathrm{S}, 70^{\circ} 24^{\prime} \mathrm{W}\right)$. Since the data from the four telescopes with main mirrors of $8 \mathrm{~m}$ in diameter are saved in a well-managed archive, spectra from VLT echelle spectrographs are a promising source for upper atmosphere research. In particular, data from the X-shooter instrument (Vernet et al., 2011) are interesting. Since 2009, it has provided spectra from 0.3 to $2.5 \mu \mathrm{m}$, which covers all $\mathrm{OH}$ bands that can be observed from the ground. This wide wavelength range is achieved by the combination of three echelle spectrographs with individual detectors (called arms), which can be operated in parallel. The resolution $\lambda / \Delta \lambda$ ranges from 3300 to 18200 depending on the arm and the selected entrance slit widths. This is sufficient to separate well the $P$-branch lines 
of the individual $\mathrm{OH}$ bands. Since the slit widths range from 0.4 to $1.6^{\prime \prime}$, depending on the arm, and the slit length is $11^{\prime \prime}$, $\mathrm{X}$-shooter observes a very small sky area. As this is compensated by the very large main mirror, even spectra with exposure times of only seconds can be used for airglow research.

In this paper, we will use high signal-to-noise $(S / N) \mathrm{X}$ shooter spectra to derive $\mathrm{OH}$ intensities, level populations, rotational temperatures, and vibrational temperatures. The 25 analysed $\mathrm{OH}$ bands allow us to study systematics and their variability in detail. The discussion will start with a description of the sample of spectra used and the basic data reduction (Sect. 2). Then, we will describe the analysis of the data in detail (Sect. 3). The results for a mean spectrum will be discussed in Sect. 4. The variability of the investigated properties will be topic of Sect. 5. Finally, we will draw our conclusions in Sect. 6.

\section{Sample selection and data reduction}

The X-shooter echelle spectrograph has been operated at Cerro Paranal since October 2009. All observed data are regularly saved in an electronic archive managed by ESO. We retrieved the archival data taken until March 2013. From April 2012, the data are incomplete due to a typical proprietary period of 1 year. We only considered spectra taken in the most frequently used, so-called stare mode, where the astronomical target is observed centred in the entrance slit (Vernet et al., 2011).

\subsection{Data reduction}

The data were reduced using the ESO public pipeline release V2.0.0 executed with the Reflex workflow V2.3 and default parameters (Modigliani et al., 2010). One type of product of this pipeline is a two-dimensional (2-D) order-merged wavelength-calibrated sky emission spectrum. It is created by a fitting procedure that excludes pixels identified as affected by the astronomical target. The pipeline sky spectrum can be collapsed into a 1-D spectrum by applying a median along the spatial direction. The resulting sky flux can be normalised to a unit sky area by considering the spatial pixel size and the variable slit width.

For a full flux calibration, it is also necessary to apply an instrumental response curve and to convert detector-related units into physical flux units. Unfortunately, the quality of the response curves produced by pipeline version V2.0.0 by observations of spectrophotometric standard stars taken with a wide $5^{\prime \prime}$ slit was insufficient. The main issue was a missing correction of atmospheric molecular absorption. This is particularly crucial in the near-IR, where strong water vapour and carbon dioxide bands cover wide wavelength ranges. With the recently released pipeline version V2.2.0, the response curve derivation has been improved significantly (Moehler et al., 2014). However, we did not re-reduce the data because it is very time consuming. Instead, we ap- plied the software tool molecfit (Smette et al., 2015; Kausch et al., 2015) to the reduced spectra of the observed spectrophotometric standard stars to fit and correct the molecular absorption by means of radiative transfer calculations. This approach should result in even better molecular absorption corrected spectra than the algorithm implemented in pipeline version V2.2.0, which is based on a library of transmission spectra.

Moreover, we created a set of master response curves excluding a large fraction of the spectrophotometric standard star observations because of an unsatisfying subtraction of the sky emission. Before May 2011, most spectra were taken in offset mode, which included a reference sky frame that was observed several minutes after the object. Since standard stars are mostly observed in the evening twilight, the strongly varying sky brightness did not allow the pipeline to subtract the sky for many cases in an adequate way. The improved observing strategy uses the nodding mode, which moves the object along the slit in order to obtain object and sky in parallel but at different positions. Although this mode results in much better sky-subtracted spectra, some observations of very faint standard stars still had to be rejected. We also neglected a few spectra taken under conditions with low atmospheric transmission, where a reliable atmospheric extinction correction based on the standard Cerro Paranal extinction curve by Patat et al. (2011) could not be performed. In the end, we could derive 12 master response curves from 56 offset and 167 nodding observations for different periods within our total period of 42 months.

Applying these master response curves should result in typical flux uncertainties of 5 to $10 \%$. However, this is no longer true for wavelengths beyond $1.9 \mu \mathrm{m}$, i.e. the $K$ band, where individual response curves can differ by factors of 2 to 3. These variations are related to the flat fields that are used to correct pixel-to-pixel sensitivity variations in the 2-D echelle spectra. The $\mathrm{X}$-shooter flat-field calibration lamps do not operate in a stable way at these long wavelengths. For this reason, the spectra of the astronomical targets and the spectrophotometric standard stars should be corrected with the same flat field. However, this is not possible if master response curves are used. As a solution, we considered 4220 pipeline-reduced spectra of so-called telluric standard stars taken during the analysed period (see also Kausch et al., 2015). These objects are usually hot stars with few lines in the near-IR. They are used to empirically derive the atmospheric molecular absorption. Since these stars are frequently observed, there is a high probability that the pipeline used a flat field taken during the daytime for an astronomical target as well as a telluric standard star. Moreover, the relatively simple spectra of telluric standard stars in the near-IR can essentially be described by the Rayleigh-Jeans law. This allowed us to derive a response correction function for a given flat field by extrapolating the flux in the affected wavelength regime for the telluric standard star spectra that were flux cal- 
ibrated with our master response curves. This approach could be successfully applied to $77 \%$ of the reduced sky spectra.

The resulting sky spectra were not corrected for atmospheric extinction, i.e. scattering and absorption, as this is different for the various components contributing to the sky emission (see Noll et al., 2012). The applied approach for the $\mathrm{OH}$ lines will be discussed in Sect. 3 .

\subsection{The sample}

For the investigation of $\mathrm{OH}$ lines, we can focus on the two $\mathrm{X}$-shooter arms VIS and NIR (Vernet et al., 2011), which cover the wavelength ranges from 0.53 to 1.02 and 0.99 to $2.48 \mu \mathrm{m}$, respectively. The full data set of sky spectra comprises 6131 VIS-arm and 9009 NIR-arm spectra. Although both arms can be operated in parallel, the number of spectra may differ, since the exposure times can be set independently and not all spectra could be reduced successfully by the Xshooter pipeline. To study all accessible $\mathrm{OH}$ bands simultaneously, we only selected combinations of VIS- and NIRarm spectra, where the exposure time and the start and end time of the exposure did not differ by more than 5 min, i.e. the typical Brunt-Väisälä period (e.g. Khomich et al., 2008). Moreover, to guarantee a reasonable $S / N$ for all selected $\mathrm{OH}$ bands (see Sect. 3), we only considered observations with exposure times of at least $3 \mathrm{~min}$ in both arms. This criterion also reduces the probability of contaminations by light profile wings of very bright astronomical objects, which have to be observed in short exposures. We also rejected a small fraction of spectra that were taken with a $5^{\prime \prime}$ wide slit. The corresponding spectral resolution is not sufficient to separate all OH $P$-branch lines well. Finally, we removed spectra with unreliable line strengths due to an erroneous data reduction. To find these spectra, we performed test measurements of $\mathrm{OH}$ rotational temperatures (see Sect. 3). For the sample of spectra already reduced by the former criteria, we only considered those observations where the temperature did not deviate by more than 3.5 standard deviations $\sigma$ from the mean value for all $\mathrm{OH}$ bands considered. $21 \%$ of the combined VIS- and NIR-arm spectra did not pass this filter.

The resulting sample consists of 343 VIS- and NIR-arm spectra and is well suited for a high-quality investigation of as many $\mathrm{OH}$ lines as possible. Figure 1 shows the time, night, and year of the selected observations. For the full period from 2009 to 2013, there is a good coverage of observing hours and days. The deviations of the corresponding averages from the mean values of an ideal distribution are only $+13 \mathrm{~min}$ and -4 days, respectively. Therefore, sample means as treated in Sect. 4 should be representative. The data also allow an analysis of nocturnal and seasonal variations (see Sect. 5) if year-to-year variations are neglected. Note that a significant fraction of the spectra was taken in a few nights with time series consisting of up to 27 exposures. Since astronomical targets are observed, zenith distances and azimuth angles are

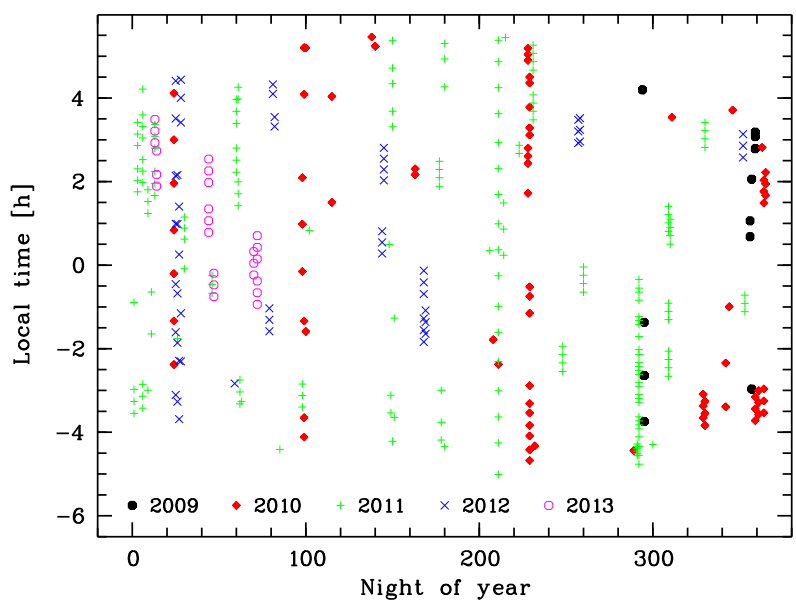

Figure 1. Sample of $343 \mathrm{X}$-Shooter observations used in this study. The local time, i.e. Universal Time (UT) corrected for the longitude of Cerro Paranal, is shown vs. the night of year. The latter is the day of year for the second half of the night. The different years of the observations are identified by different symbols and colours (see legend at the bottom of the figure).

variable. The mean zenith distance for the sample is $36^{\circ}$ with a scatter of $11^{\circ}$.

As discussed in Sect. 2.1, the flux calibration is relatively uncertain in the photometric $K$ band. For studying the $\mathrm{OH}$ bands (8-6) and (9-7), we therefore selected a subsample of the 343 observations which only contains spectra where a flux correction based on telluric standard stars could be performed. Moreover, we had to further reduce this sample due to the use of a so-called $K$-blocking filter (Vernet et al., 2011) for 36 observations. This filter avoids the light from the $K$ band that scatters into shorter wavelengths, which significantly reduces the instrument-related continuum flux. However, the use of this filter limits the reliable wavelength range to wavelengths below $2.1 \mu \mathrm{m}$, which especially affects $\mathrm{OH}(9$ 7). Thus, we will also use a sample of 240 spectra, which provides good quality data for the two reddest, accessible $\mathrm{OH}$ bands. The average time and date for this sample differ from the ideal mean values by $32 \mathrm{~min}$ and 6 days, respectively. Therefore, this $K$-band sample can also be considered as representative of Cerro Paranal for the covered period.

\section{Analysis}

In the following, we will describe the selection of suitable $\mathrm{OH}$ lines (Sect. 3.1), the measurement of their intensities (Sect. 3.2), and the derivation of rotational and vibrational temperatures (Sects. 3.3 and 3.4).

\subsection{Line selection}

The VIS- and NIR-arm X-shooter spectra cover all $\mathrm{OH}$ bands between 0.53 and $2.48 \mu \mathrm{m}$, i.e. 29 bands with $v^{\prime} \leq 9$. Except for $\mathrm{OH}(7-1)$, the $P$-branch lines of these bands are shown 
in Fig. 2 for a spectrum of the median pixel intensities of the $K$-band sample discussed in Sect. 2.2. It is not possible to derive reliable line intensities and temperatures for all bands. In the case of $\mathrm{OH}(7-1)$ and $\mathrm{OH}(5-0)$, the faintness of the lines prevents trustworthy measurements, even with exposure times of at least $3 \mathrm{~min}$. Moreover, they are in the optical wavelength range, where the sky continuum can be relatively strong (e.g. scattered moonlight or zodiacal light, see e.g. Noll et al., 2012 and Jones et al., 2013), which makes the line integration highly uncertain. Therefore, we did not consider these bands. The faintest remaining band is $\mathrm{OH}(8-2)$. We also rejected $\mathrm{OH}(9-6)$ and $\mathrm{OH}(7-5)$ because they are located in wavelength ranges that are highly affected by strong water vapour absorption bands (e.g. Smette et al., 2015). Since there are not at least three lines with low absorption, a reliable rotational temperature measurement is not possible. Hence, our study comprises $25 \mathrm{OH}$ bands.

With a minimum $\lambda / \Delta \lambda$ of 5400 in the VIS arm and 3000 in the NIR arm for the selected X-shooter spectra, the $P$ branches (related to the total angular momentum change $J^{\prime \prime}=J^{\prime}+1$ ) of all OH bands are well resolved (see Fig. 2), i.e. lines of the same band are separated. As this criterion is not fulfilled for the $R\left(J^{\prime \prime}=J^{\prime}-1\right)$ and $Q$-branches $\left(J^{\prime \prime}=\right.$ $J^{\prime}$ ), we will neglect them in the following (except for a few lines discussed below). $\mathrm{OH}$ lines originating from high rotational levels are not suitable for the derivation of rotational temperatures, since these levels are strongly overpopulated compared to the expected distribution for a typical ambient temperature in the mesopause of about $200 \mathrm{~K}$ (Pendleton et al., 1989, 1993; Perminov and Semenov, 1992; Cosby and Slanger, 2007). Only for the first few rotational levels can a population distribution close to a rotational-translational equilibrium be expected. Therefore, following Cosby and Slanger (2007), we only consider $\mathrm{OH}$ lines originating from rotational levels $N^{\prime} \leq 4$ of both electronic substates $\mathrm{X}^{2} \Pi_{3 / 2}$ $(i=1)$ and $\mathrm{X}^{2} \Pi_{1 / 2}(i=2)$, which are caused by a coupling of the electron spin and the orbital angular momentum (e.g. Rousselot et al., 2000; Khomich et al., 2008). For the $P$ branch, these $P_{i^{\prime}}\left(N^{\prime}\right)$ lines are $P_{1}(1)$ to $P_{1}(4)$ for $\mathrm{X}^{2} \Pi_{3 / 2}$ and $P_{2}$ (1) to $P_{2}$ (4) for $\mathrm{X}^{2} \Pi_{1 / 2}$. Intercombination lines between the two electronic substates were neglected due to their extremely low intensity (e.g. Mies, 1974; Goldman et al., 1998).

As Fig. 2 indicates, the number of selected $P$-branch lines of an $\mathrm{OH}$ band is often lower than eight. Individual lines had to be mainly rejected due to low atmospheric transmission and blending with lines not belonging to the same band. A preliminary identification of affected lines was performed by means of a high-resolution atmospheric transmission spectrum and an airglow line list optimised for Cerro Paranal (Noll et al., 2012, 2014). The transmission spectrum was calculated by using the Line-By-Line Radiative Transfer Model (LBLRTM, Clough et al., 2005) for an average atmospheric temperature and molecular abundance profile of Cerro Paranal. The line list was derived from observations at
Cerro Paranal (Hanuschik, 2003; Cosby et al., 2006; Patat, 2008) and theoretical data (Goldman et al., 1998; Rousselot et al., 2000). In a second step, we evaluated the quality of the $\mathrm{OH}$ line data by means of preliminary measurements of rotational temperatures $T_{\text {rot }}$ (Sect. 3.3) for the transmissioncorrected and sample-averaged line intensities (Sect. 3.2). In order to identify critical lines, $T_{\text {rot }}$ were derived for all kinds of line subsets in each band. In particular, $T_{\text {rot }}$ from only two lines were sensitive to outliers. Finally, we classified $152 P$ branch lines as reliable. Seven additional lines (see Fig. 2) were considered to be good enough for a derivation of $T_{\text {rot }}$ corrections depending on the line set (see Sect. 3.3).

After the rejection of unreliable lines, only two $P$-branch lines were left for $\mathrm{OH}(6-4)$, which does not allow the determination of $T_{\text {rot }}$ uncertainties. For this reason, we added the relatively strong $R$-branch line $R_{1}$ (2), which is not blended by other lines in the $\mathrm{X}$-shooter spectra and not affected by low atmospheric transmission. To compare the $T_{\text {rot }}$ derived from $\mathrm{OH}(6-4)$ with those of the other bands (see Sect. 4.2), we could also measure $R_{1}(2)$ in the $\mathrm{OH}$ bands (3-0), (3-1), (5-2), (5-3), and (9-7).

\subsection{Derivation of line intensities}

The $\mathrm{OH}$ line intensities were measured for each spectrum by a fully automatic procedure.

First, the sky continuum was roughly determined by applying a median filter to the pixel intensities. The filter was about an order of magnitude wider than the pixel ranges affected by single airglow lines. The resulting continuum was then subtracted from the spectrum. For most $\mathrm{OH}$ bands, the continuum is relatively faint (see Fig. 2), i.e. inaccuracies in the derived continuum only have a minor impact. Moreover, the continuum tends to be relatively smooth at the positions of the selected lines. Lines with critical continua in most sample spectra were rejected in the course of the selection procedure described in Sect. 3.1.

The line intensities were integrated in wavelength ranges which had a width of about 3 times the line FWHM. Hence, the integration limits had to be modified as a function of the line wavelength and the spectral resolution, which depends on X-shooter arm and slit width. We also considered that any measured $\mathrm{OH}$ line consists of two slightly separated $\Lambda$ doublet lines (e.g. Rousselot et al., 2000). However, this was only a minor correction. For the most frequently used $0.9^{\prime \prime}$ slit (52\% of the sample spectra), even the separation of the $P_{1}$ (4) line doublet was always lower than $4 \%$ of the integration range. The line integrations were performed for two different sets of line wavelengths. We used the HITRAN2012 line database (Rothman et al., 2013), where the $\mathrm{OH}$ data are based on Goldman et al. (1998) (see Rothman et al., 2009). We also performed our analysis for an alternative (and probably the most recent) set of $\mathrm{OH}$ line data calculated by van der Loo and Groenenboom $(2007,2008)$. The two different sets allow us to investigate the effect of deviations in the roto- 

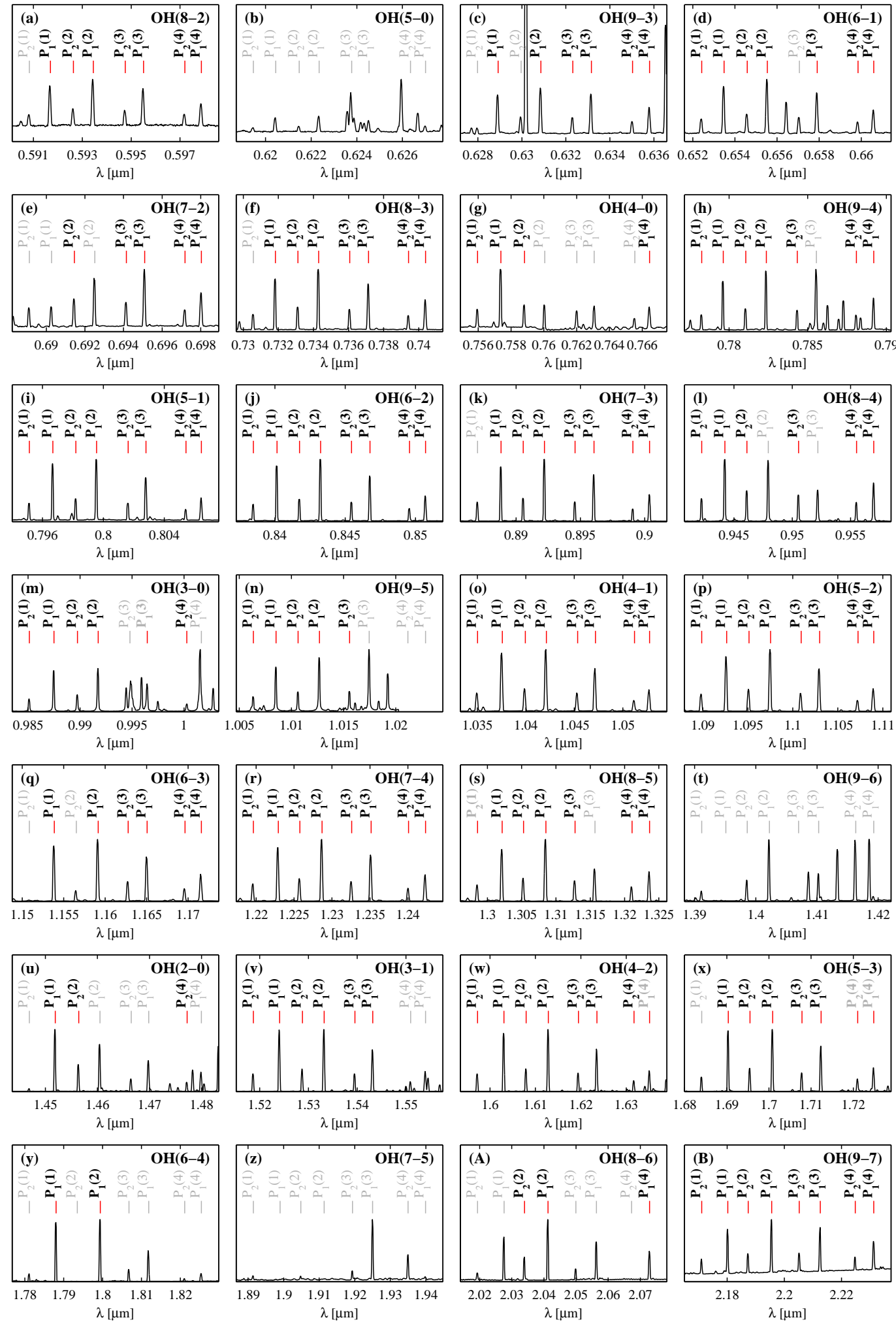

Figure 2. $P$-branch lines of the $\mathrm{OH}$ bands used in this study. The displayed spectrum is the pixel-related median of the $240 \mathrm{X}$-shooter VISand NIR-arm observations which cover all bands and where a reliable flux calibration could be performed in the $K$ band. Lines with black labels were used for the derivation of temperatures. Lines with grey labels were excluded from this study. An exception are those with red marker lines. They were considered for the $T_{\text {rot }}$ correction due to differences in the sets of lines available for each band. 
vibrational level energies and Einstein coefficients on $\mathrm{OH}$ level populations and temperatures. In the following, we will call them G98 and LG08, respectively. To set the integration ranges, we took the average of the $\Lambda$ doublet wavelengths, which is reasonable, since the intensities of the doublet lines are almost identical. Contaminations by other lines in the integration ranges should be negligible due to our careful line selection procedure (Sect. 3.1), which also included a special check for observations taken with a wide slit $\left(>1^{\prime \prime}\right)$, i.e. low resolution.

Since astronomical targets are observed at different zenith distances $z$, we converted the measured line intensities $I(z)$ into those for the zenith $I(0)$ by assuming a homogeneous $\mathrm{OH}$ emission layer at a height $h$ of $87 \mathrm{~km}$ (e.g. Baker and Stair, 1988) and applying

$I(0)=I(z) \sqrt{1-\left(\frac{R \sin (z)}{R+h}\right)^{2}}$

(van Rhijn, 1921), where $R$ is the Earth's radius. The effect of the altitude distribution of the emission with a typical FWHM of $8 \mathrm{~km}$ (e.g. Baker and Stair, 1988) on the correction is negligible $(<1 \%)$, since the largest zenith distance in our sample is only $65^{\circ}$.

Since the molecular absorption in the lower atmosphere can be strong in the near-IR, a significant fraction of the $\mathrm{OH}$ lines cannot be used (see Sect. 3.1). We tried to reduce the number of rejections by correcting for the absorption, which is promising for low optical depths. For this purpose, we used a high-resolution $\left(\lambda / \Delta \lambda \approx 10^{6}\right)$ transmission spectrum for Cerro Paranal (Noll et al., 2012) calculated by LBLRTM (Clough et al., 2005) for the zenith and an amount of precipitable water vapour (PWV) of $2.5 \mathrm{~mm}\left(=\mathrm{PWV}_{\text {ref }}\right)$. Here, also the wavelength-dependent fraction of water vapour absorption $f_{\mathrm{H}_{2} \mathrm{O}}$ was known. The chosen $\mathrm{PWV}_{\text {ref }}$ is close to the sample median and mean of 2.3 and $3.1 \mathrm{~mm}$, respectively. We also assumed that the $\mathrm{OH}$ lines have a Gaussian line profile due to Doppler broadening related to a temperature of $200 \mathrm{~K}$, which results in FWHM of a few pm (cf. Noll et al., 2012). The normalised profiles were then multiplied by the transmission spectrum to obtain reference transmission values for each $\Lambda$ doublet line. In order to adapt these values to the real observing conditions, we calculated the $z$-dependent air mass $X$ relevant for the molecular absorption by means of

$X=\left(\cos (z)+0.025 \mathrm{e}^{-11 \cos (z)}\right)^{-1}$

(Rozenberg, 1966), where $X$ converges to 40 at the horizon. The PWV values were primarily taken from the molecfit (Smette et al., 2015; Kausch et al., 2015) fitting results for the sample of telluric standard stars discussed in Sect. 2.1. Moreover, we considered measurements from the ESO PWV monitor for Cerro Paranal, which are based on different instruments and methods. Since there are systematic differences in the PWV from the different data sets, we derived correction factors from the sample means and took the molecfit
VIS-arm results as a reference. For four nights without PWV measurements, we used the PWV values of the model-based molecfit atmospheric input profiles (see Smette et al. (2015) for their time-dependent creation and Kausch et al. (2015) for their accuracy). The reference transmission curve $T_{\text {ref }}$ was then adapted by the approximation

$T=T_{\text {ref }}\left(1+\frac{{ }^{P W V}-\mathrm{PWV}_{\text {ref }}}{\mathrm{PWV}_{\text {ref }}} f_{\mathrm{H}_{2} \mathrm{O}}\right) X$.

Finally, the mean transmission of the two lines of each $\Lambda$ doublet was calculated to correct the measured line intensities. This approach allowed us to significantly increase the number of $\mathrm{OH}$ lines, since only 88 of the 153 selected lines had an average transmission of at least $99 \%$ for $\mathrm{G} 98 \mathrm{OH}$ data. Lines were included with average transmissions down to $77 \%\left(\mathrm{OH}(2-0) P_{1}(1)\right)$. In particular, the corrections made it possible to study the strongly affected $\mathrm{OH}$ bands (2-0), (6$3),(6-4)$, and (8-4). For this, an accuracy of the line absorption correction of 10 to $20 \%$ had to be achieved.

We did not perform an intensity correction for the extinction by scattering, as this should be very small due to the relatively long wavelengths of the $\mathrm{OH}$ lines and the very low aerosol column densities at Cerro Paranal (Patat et al., 2011; Jones et al., 2014). Moreover, the emission distribution over the entire sky makes the extinction losses much smaller than for point sources (Chamberlain, 1961; Noll et al., 2012). Nevertheless, we cannot exclude that some observations were made through thin cirrus clouds. Even in this case, the $\mathrm{OH}$ rotational temperatures based on lines with small wavelength differences should still be useful (e.g. Noll et al., 2014).

\subsection{Derivation of rotational temperatures}

Assuming a Boltzmann distribution for the population of rotational levels of an $\mathrm{OH}$ band, i.e. LTE, a rotational temperature $T_{\text {rot }}$ can be derived from the slope of a regression line for

$$
\begin{aligned}
y= & \ln \left(\frac{I\left(i^{\prime}, v^{\prime}, N^{\prime} \rightarrow i^{\prime \prime}, v^{\prime \prime}, N^{\prime \prime}\right)}{g^{\prime}\left(i^{\prime}, N^{\prime}\right) A\left(i^{\prime}, v^{\prime}, N^{\prime} \rightarrow i^{\prime \prime}, v^{\prime \prime}, N^{\prime \prime}\right)}\right) \\
= & \text { const }-\frac{h c E^{\prime}\left(i^{\prime}, v^{\prime}, N^{\prime}\right)}{k T_{\text {rot }}}
\end{aligned}
$$

as a function of the energy $E^{\prime}$ (as wave number) of the electronic, vibrational, and rotational levels $i^{\prime}, v^{\prime}$, and $N^{\prime}$ of the upper state (Meinel, 1950b; Mies, 1974). The constants $h$, $c$, and $k$ are the Planck constant, speed of light, and Boltzmann constant, respectively. Apart from $E^{\prime}$, the required input for the $T_{\text {rot }}$ derivation is the line intensity $I$, the statistical weight of the upper state $g^{\prime}=2\left(2 J^{\prime}+1\right)$ depending on the angular momentum $J^{\prime}$, and the Einstein coefficient $A$. The term $I / g^{\prime} A$ is proportional to the population of a hyperfinestructure level. While $I$ is measured, $E^{\prime}$ and $A$ are taken either from G98 or LG08 (see Sect. 3.2). Since the $\mathrm{OH} \Lambda$ 


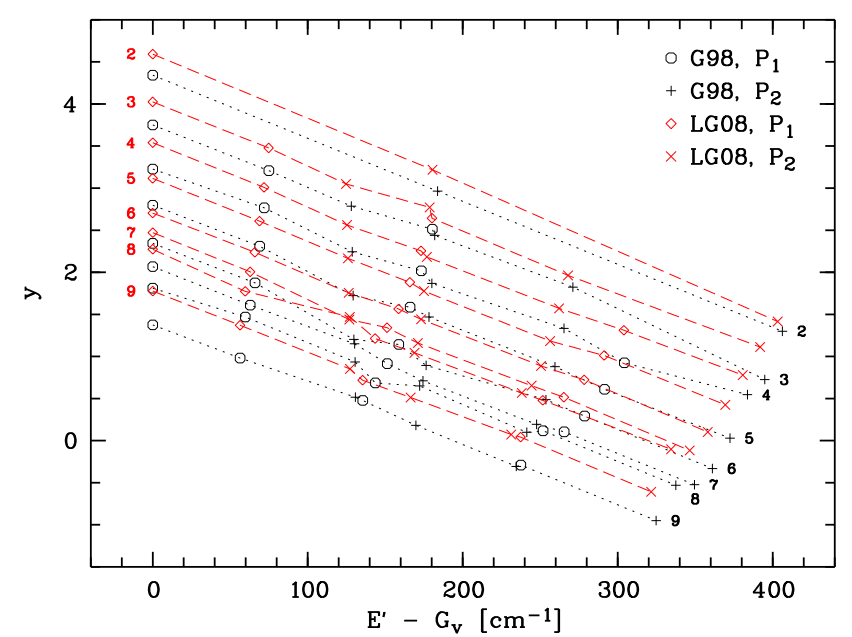

Figure 3. Derivation of $\mathrm{OH} T_{\text {rot }}$ under the assumption of a Boltzmann distribution for the different rotational upper levels. The abscissa shows the energy of a given level relative to the lowest rotational level $G_{v}$ of a band in $\mathrm{cm}^{-1}$. The ordinate displays the natural logarithm of the population of a hyperfine-structure level relative to 1 Rayleigh second. The data points indicate the mean values of $E^{\prime}-G_{v}$ and $y$ derived from the sample mean intensities for the selected $\mathrm{OH}$ lines with the same $v^{\prime}$. The different symbols and colours distinguish lines of the $P_{1}$ - and $P_{2}$-branches as well as the use of energy values and Einstein coefficients of G98 and LG08, respectively (see legend). The dotted (G98) and dashed lines (LG08) connect OH lines with the same $v^{\prime}$ (see labels).

doublets are not resolved (see Sect. 3.2), $I$ is the sum of the intensities of both doublet lines. For this reason, the $A$ coefficients, which are identical for G98, were also summed and both (very similar) $E^{\prime}$ were averaged.

For illustration, Fig. 3 shows the averaged $y$ and $E^{\prime}$ for the different $P$-branch lines, upper vibrational levels $v^{\prime}$, and molecular line data sets (G98 and LG08) derived from the mean line intensities of the sample. The $y$ values of lines with the same upper state (which indicated a typical scatter of 0.1 ) were averaged to improve the clarity of the plot. The level energy $E^{\prime}$ is given relative to the lowest energy level of a band $G_{v}$, i.e. $P_{1}(1)$ is always located at 0 . For the other lines, $E^{\prime}-G_{v}$ depends on $v^{\prime}$. The line data source plays a minor role. The highest energy state of each $v^{\prime}$ is related to $P_{2}$ (4). The $y$ values decrease with increasing $v^{\prime}$, which implies a lower relative population of higher vibrational levels. The $y$ values also indicate significant differences in the Einstein coefficients of the G98 and LG08 line data sets. The figure demonstrates that data points with the same $v^{\prime}$ can be well fitted by a straight line.

Since the resulting $T_{\text {rot }}$ for the different $\mathrm{OH}$ bands are based on different line sets (see Fig. 2), a comparison might fail due to systematic offsets depending on the selected lines (see Sect. 4.1). Therefore, all $T_{\text {rot }}$ measurements were corrected to be representative of a reference line set consisting of the first three $P_{1}$ - and $P_{2}$-branch lines. For this, we calcu- lated $T_{\text {rot }}$ from the mean line intensities of the sample for all relevant line combinations. We only considered the 16 most reliable bands for these calculations. Very faint bands $(\mathrm{OH}(8-2), \mathrm{OH}(9-3), \mathrm{OH}(6-1), \mathrm{OH}(7-2)$, and $\mathrm{OH}(4-0))$ and those strongly affected by atmospheric absorption $\mathrm{OH}(6-$ $3), \mathrm{OH}(2-0), \mathrm{OH}(6-4)$, and $\mathrm{OH}(8-6))$ were not used. For the selected $\mathrm{OH}$ bands, we also included some acceptable extra lines (see Sect. 3.1) to increase the number of bands for certain line sets. Then, for the correction, the average $\Delta T_{\text {rot }}$ relative to the reference line set was derived for each required line combination. This approach neglects a possible $v^{\prime}$ dependence of the $\Delta T_{\text {rot }}$, which is hard to derive due to the limited number of bands and the required very high accuracy in $T_{\text {rot }}$. However, this would only be a second order correction, as the change in $E^{\prime}-G_{v}$ for different $v^{\prime}$ but fixed rotational transition is smaller than the corresponding change for different lines and fixed $v^{\prime}$ (see Fig. 3). Although the corrections were calculated for the sample mean, we also applied them to the $T_{\text {rot }}$ for the individual spectra. The $\Delta T_{\text {rot }}$ do not appear to significantly depend on time (see Sect. 4.1).

For the $T_{\text {rot }}$ derived from mean intensities (Sect. 4.2), we also corrected for the smaller sample of spectra suitable for the $\mathrm{OH}$ bands (8-6) and (9-7) (see Sect. 2.2). The $T_{\text {rot }}$ of the two bands were measured for the mean intensities of the $K$ band sample. This was also done for the bands with the same $v^{\prime}$ but different $v^{\prime \prime}$. For the latter, we could derive the $\Delta T_{\text {rot }}$ for the two samples. The mean difference for each $v^{\prime}$ was then applied to the corresponding $\mathrm{OH}$ band in the $K$ band. The resulting correction for $\mathrm{G} 98$ parameters was $0.0 \mathrm{~K}$ for $\mathrm{OH}(9-7)$ and $+1.1 \mathrm{~K}$ for $\mathrm{OH}(8-6)$. For LG08, the corresponding values were -0.1 and $+1.0 \mathrm{~K}$, respectively.

Apart from the $T_{\text {rot }}$ results for each $\mathrm{OH}$ band, we will also discuss mean values for all bands with the same $v^{\prime}$, i.e. the same roto-vibrational upper states. For the discussion of the sample means in Sect. 4, we considered all 25 bands for the $v^{\prime}$-related $T_{\text {rot }}$ averaging. For the derivation of the mean values for the individual observations in Sect. 5.2, we neglected $\mathrm{OH}(8-2)$ (see Fig. 2), which is the band with the lowest $S / N$. This measure made the $v^{\prime}=8$ average distinctly more robust. Excluding $\mathrm{OH}(8-2)$ would change $T_{\text {rot }}\left(v^{\prime}=8\right)$ for the full sample by $+0.3 \mathrm{~K}$ for $\mathrm{G} 98$ and $+1.9 \mathrm{~K}$ for LG08. Therefore, we subtracted these amounts from the $T_{\text {rot }}\left(v^{\prime}=8\right)$ for the individual observations.

\subsection{Derivation of vibrational temperatures}

The approach for deriving $T_{\text {rot }}$ discussed in Sect. 3.3 can also be used for obtaining a temperature for the population of levels belonging to different $\mathrm{OH}$ bands with deviating $v^{\prime}$. Since the mean $y$ value of a band strongly depends on the selected lines (see Fig. 3), we measured vibrational temperatures $T_{\text {vib }}$ by extrapolating the $y$ value of each line to the energy of the $v^{\prime}$-dependent ground state $G_{v}$, where $P_{1}$ (1) originates. This is possible by using the slopes related to the $T_{\text {rot }}$ measurements. In fact, we used the $v^{\prime}$-averaged $T_{\text {rot }}$ (see Sect. 3.3) 


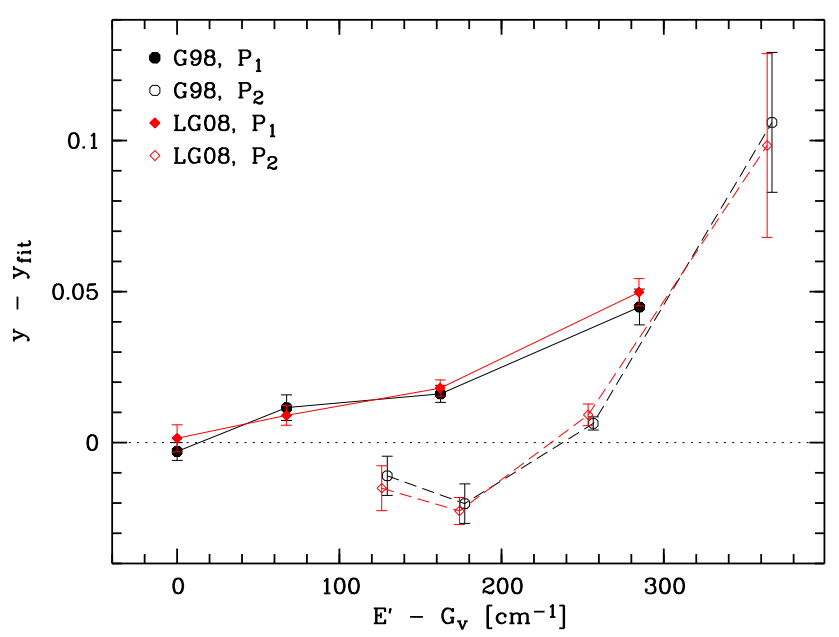

Figure 4. Over- and underpopulation of the upper rotational levels of the $P$-branch $\mathrm{OH}$ lines relative to a regression line based on the first three $P_{1}$ and $P_{2}$ lines. The abscissa shows the energy difference in $\mathrm{cm}^{-1}$ of an upper rotational level $E^{\prime}$ to the lowest level $G_{v}$. The ordinate exhibits the natural logarithm of a population ratio. The data points show the mean values and mean errors of the four investigated $P_{1}$ (filled symbols and solid lines) and $P_{2}$ lines (open symbols and dashed lines) for the $\mathrm{OH}$ bands (4-1), (5-2), (6-2), and (7-4). The results for the G98 and LG08 line parameters are marked by circles and diamonds, respectively.

for the sample means (Sect. 4) as well as the individual spectra (Sect. 5.2) and applied the corresponding shifts in $y$. The regression line for the $T_{\mathrm{vib}}$ derivation was then calculated for the corrected $y$ values vs. the $v^{\prime}$-related $G_{v}$ of the selected $\mathrm{OH}$ lines. $T_{\text {vib }}$ were derived for different $v^{\prime}$ ranges (see Sects. 4.3 and 5).

\section{Results for mean line intensities}

Studying systematics in the roto-vibrational level populations and the corresponding $T_{\text {rot }}$ and $T_{\text {vib }}$ requires highquality data. Although the selection of our X-shooter data set of 343 spectra ( 240 spectra for the $K$ band) was already very restrictive in this respect (Sect. 2.2), the statistical and systematic uncertainties can be further reduced by analysing the mean intensities of $\mathrm{OH}$ lines. For this reason, we will start with a discussion of results obtained for the sample mean. We will describe the influence of different line sets (Sect. 4.1) and $\mathrm{OH}$ bands (Sect. 4.2) on $T_{\text {rot }}$ and discuss vibrational level populations and temperatures (Sect. 4.3). Time-dependent variations will be treated in Sect. 5 based on the individual measurements.

\subsection{Rotational level populations and temperatures for different line sets}

The general population of the different roto-vibrational $\mathrm{OH}$ levels for the sample mean intensities was discussed in Sect. 3.3 (see Fig. 3). However, we still need to validate the assumption that the first four lines of the $P_{1}$ and $P_{2}$-branches show a Boltzmann-like intensity distribution related to the ambient temperature (e.g. Beig et al., 2003; Perminov et al., 2007; Cosby and Slanger, 2007). For this purpose, we subtracted the regression fit from the first three $P_{1}$ and $P_{2}$ lines (our reference set) $y_{\text {fit }}$ from the $y$ of the different lines for the four highly reliable intermediate- $v^{\prime} \mathrm{OH}$ bands (4-1), (52 ), (6-2), and (7-4), where all eight $P$-branch lines could be measured. The resulting $\Delta y=y-y_{\text {fit }}$ of each line were then averaged.

Figure 4 displays the mean $\Delta y$ as a function of the mean energy difference $E^{\prime}-G_{v}$. The $\Delta y$ distribution shows a significant deviation from the zero line. The line with the highest energy difference $P_{2}(4)$ indicates the largest deviation, which corresponds to a relative overpopulation of about $10 \%$ for the $N^{\prime}=4$ rotational level of the $\mathrm{X}^{2} \Pi_{1 / 2}$ substate. The $\Delta y$ of $P_{1}$ (4) implies an overpopulation of about $5 \%$ for $N^{\prime}=4$ of $X^{2} \Pi_{3 / 2}$. All other lines have smaller deviations of about $2 \%$ in maximum. Interestingly, for a given level energy, $X^{2} \Pi_{1 / 2}$ levels tend to have a several per cent lower population than $X^{2} \Pi_{3 / 2}$ levels. This pattern could also be confirmed for different band selections as the preferred four bands. A significant trend with $v^{\prime}$ could not be detected. The $\Delta y$ do not appear to depend on the molecular line data, since the results for G98 and LG08 line data do not show any significant differences. For this reason, the deviations from the zero line appear to be very reliable. Moreover, we checked the variability of the $\Delta y$ pattern observed by also performing the analysis for the individual observations. There was a remarkable stability. For $P_{2}(4)$, we only found a scatter in $\Delta y$ of about 0.02. All other lines showed variations of less than about 0.01 .

The results for the $N^{\prime}=4$ lines confirm the trend of increasing deviation from the LTE for increasing $N^{\prime}$, which was already convincingly shown by Pendleton et al. (1993). The incomplete rotational-translational equilibration of $N^{\prime}=4$ levels suggests that only $N^{\prime} \leq 3$ lines should be used for estimates of the mesopause temperature, which would be more restrictive than the $N^{\prime} \leq 5$ criterion reported by Beig et al. (2003) referring to Perminov and Semenov (1992). Nevertheless, we used all measured lines for our $T_{\text {rot }}$ derivations (Sect. 3.3), since this makes the regression fit more robust, especially if several low- $N^{\prime}$ lines are rejected (Sect. 3.1). In this case, a correction of the $T_{\text {rot }}$ is required to avoid unrealistic mesopause temperatures (see Sect. 3.3). To illustrate the deviations, Fig. 5 shows $\Delta T_{\text {rot }}$ for different line sets relative to our reference set consisting of the six $N^{\prime} \leq 3$ lines. For the determination, we again used the $\mathrm{OH}$ bands (4-1), (5-2), (6-2), and (7-4). Using the eight $P$-branch 
Table 1. Measured and corrected rotational temperatures in $\mathrm{K}$ from different $\mathrm{OH}$ bands for G98 and LG08 line parameters.

\begin{tabular}{|c|c|c|c|c|c|c|c|c|}
\hline $\mathrm{OH}\left(v^{\prime}-v^{\prime \prime}\right)$ & Line set ${ }^{\mathrm{a}}$ & Fig. 2 & $\begin{array}{l}T_{\text {meas }} \\
\text { (G98) }\end{array}$ & $\begin{array}{r}\Delta T_{\text {corr }}^{\mathrm{b}} \\
(\mathrm{G} 98)\end{array}$ & $\begin{array}{c}T_{\text {ref }}^{\mathrm{b}} \\
(\mathrm{G} 98)\end{array}$ & $\begin{array}{c}T_{\text {meas }} \\
\text { (LG08) }\end{array}$ & $\begin{array}{c}\Delta T_{\text {corr }}^{\mathrm{b}} \\
(\mathrm{LG} 08)\end{array}$ & $\begin{array}{c}T_{\mathrm{ref}}^{\mathrm{b}} \\
\text { (LG08) }\end{array}$ \\
\hline $\mathrm{OH}(2-0)$ & 001100010 & (u) & 192.1 & -4.4 & 187.7 & 182.4 & -4.0 & 178.4 \\
\hline $\mathrm{OH}(3-0)$ & 011110010 & (m) & 192.8 & -4.3 & 188.5 & 190.3 & -4.0 & 186.3 \\
\hline $\mathrm{OH}(3-1)$ & 011111100 & (v) & 195.3 & 0.0 & 195.3 & 195.2 & 0.0 & 195.2 \\
\hline $\mathrm{OH}(4-0)$ & 011100001 & (g) & 198.4 & -3.4 & 195.0 & 194.7 & -5.5 & 189.3 \\
\hline $\mathrm{OH}(4-1)$ & 011111111 & (o) & 201.1 & -4.5 & 196.6 & 198.2 & -5.0 & 193.2 \\
\hline $\mathrm{OH}(4-2)$ & 011111110 & (w) & 197.6 & -3.8 & 193.8 & 195.5 & -3.5 & 192.0 \\
\hline $\mathrm{OH}(5-1)$ & 011111111 & (i) & 198.7 & -4.5 & 194.2 & 194.4 & -5.0 & 189.4 \\
\hline $\mathrm{OH}(5-2)$ & 011111111 & (p) & 197.7 & -4.5 & 193.2 & 193.2 & -5.0 & 188.2 \\
\hline $\mathrm{OH}(5-3)$ & 001111100 & (x) & 195.5 & -0.1 & 195.3 & 194.1 & -0.1 & 194.0 \\
\hline $\mathrm{OH}(6-1)$ & 011110111 & (d) & 198.9 & -5.0 & 193.9 & 192.6 & -5.6 & 187.1 \\
\hline $\mathrm{OH}(6-2)$ & 011111111 & (j) & 202.4 & -4.5 & 197.9 & 198.4 & -5.0 & 193.4 \\
\hline $\mathrm{OH}(6-3)$ & 001011111 & (q) & 199.0 & -4.1 & 194.9 & 194.2 & -4.5 & 189.7 \\
\hline $\mathrm{OH}(6-4)$ & 101010000 & (y) & 189.9 & +9.7 & 199.6 & 189.1 & +7.1 & 196.2 \\
\hline $\mathrm{OH}(7-2)$ & 000101111 & (e) & 201.8 & -10.4 & 191.4 & 194.1 & -9.9 & 184.2 \\
\hline $\mathrm{OH}(7-3)$ & 001111111 & (k) & 198.4 & -4.2 & 194.1 & 193.3 & -4.6 & 188.7 \\
\hline $\mathrm{OH}(7-4)$ & 011111111 & (r) & 200.9 & -4.5 & 196.4 & 198.9 & -5.0 & 193.9 \\
\hline $\mathrm{OH}(8-2)$ & 001111111 & (a) & 207.4 & -4.2 & 203.1 & 203.1 & -4.6 & 198.6 \\
\hline $\mathrm{OH}(8-3)$ & 001111111 & (f) & 205.4 & -4.2 & 201.2 & 200.3 & -4.6 & 195.7 \\
\hline $\mathrm{OH}(8-4)$ & 011101011 & (1) & 203.2 & -5.0 & 198.1 & 203.8 & -5.3 & 198.5 \\
\hline $\mathrm{OH}(8-5)$ & 001111011 & (s) & 208.4 & -4.2 & 204.2 & 203.4 & -4.6 & 198.9 \\
\hline $\mathrm{OH}(8-6)^{\mathrm{c}}$ & 000110001 & (A) & 207.5 & -4.2 & 203.3 & 219.2 & -10.0 & 209.2 \\
\hline $\mathrm{OH}(9-3)$ & 001011111 & (c) & 199.7 & -4.1 & 195.6 & 193.2 & -4.5 & 188.7 \\
\hline $\mathrm{OH}(9-4)$ & 011111011 & (h) & 203.5 & -4.5 & 199.0 & 200.0 & -4.9 & 195.1 \\
\hline $\mathrm{OH}(9-5)$ & 011111000 & (n) & 195.3 & 0.0 & 195.3 & 190.1 & 0.0 & 190.0 \\
\hline $\mathrm{OH}(9-7)^{\mathrm{c}}$ & 011111111 & (B) & 202.4 & -4.6 & 197.8 & 198.0 & -5.1 & 192.9 \\
\hline
\end{tabular}

lines already causes a temperature excess of 6 to $7 \mathrm{~K}$ independent of the choice of G98 or LG08 data. If only the four lines with the highest $E^{\prime}-G_{v}$ are used, a deviation of about $20 \mathrm{~K}$ is reached. The latter is already about $10 \%$ of a typical mesopause temperature. We could not find a significant temporal variation in the relative rotational level populations. Therefore, a $T_{\text {rot }}$ time series that always uses the same set of lines (see Beig et al., 2003, for an overview) should only be offset by a nearly constant $\Delta T_{\text {rot }}$.

Even if the $N^{\prime}=4$ levels are neglected, there are significant deviations in the relative level populations (Fig. 4), which can affect the $T_{\text {rot }}$ especially if the number of measured lines is small (Fig. 5). In particular, the level population differences for the two electronic substates are striking. Both states do not seem to be in equilibrium due to insufficient relaxation processes by collisions and radiation. This could allow signatures of the nascent level population related to the $\mathrm{OH}$ creation to survive (cf. Sect. 4.2). Without the lines $P_{2}$ (1) to $P_{2}$ (3), the resulting $T_{\text {rot }}$ would be about $3 \mathrm{~K}$ higher than for all $N^{\prime} \leq 3$ lines if the $\mathrm{OH}$ bands of Fig. 5 are considered. It is not clear whether these $P_{1}$-based temperatures would be more realistic.

\subsection{Rotational temperatures for different $\mathrm{OH}$ bands}

Applying the procedure described in Sect. 3.3, we derived corrected $T_{\text {rot }}$ for the reference set of $N^{\prime} \leq 3 P$-branch lines for the 25 measured $\mathrm{OH}$ bands listed in Table 1 (see also Fig. 2). Table 1 and Fig. 6 show the sample mean results for the molecular line data sets of G98 and LG08. Since the upper levels of lines from $\mathrm{OH}$ bands with fixed $v^{\prime}$ are the same, we also display the mean $T_{\text {rot }}$ for each $v^{\prime}$ in Fig. 6 . The plotted mean errors were primarily derived from the variance of the differences between the band- and $v^{\prime}$-related $T_{\text {rot }}$. Since the uncertainties in the individual $T_{\text {rot }}$ can be quite different, we also considered the uncertainties of the regression analysis and $T_{\text {rot }}$ correction. As these errors are unrealistically high due to systematic deviations in the rotational level populations (see Sect. 4.1), we scaled them by a global factor ( 0.42 and 0.53 for G98 and LG08 data) derived from the first error derivation approach and calculated $v^{\prime}$-related mean errors. If these errors were higher than those related to the $T_{\text {rot }}$ differences, we used them instead. However, this was only true in a few cases and the change was moderate. The resulting mean errors for $T_{\text {rot }}\left(v^{\prime}\right)$ are between 1.0 and $2.0 \mathrm{~K}$ for G98 and 1.8 and $3.6 \mathrm{~K}$ for LG08. The true uncertainties might even be smaller if bands with the same $v^{\prime}$ showed different $T_{\text {rot }}$ for physical reasons. Otherwise the differences 


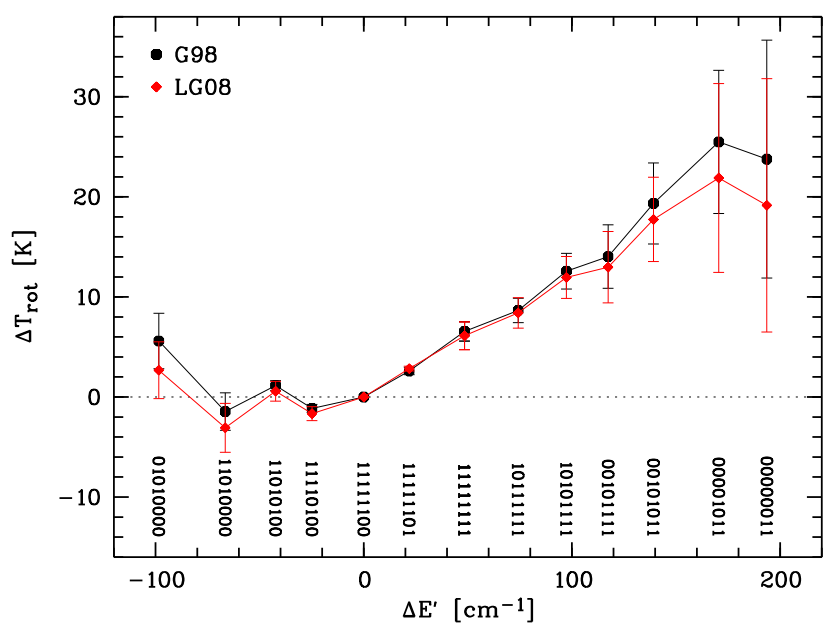

Figure 5. Change in $T_{\text {rot }}$ by differences in the set of lines used for its determination. The difference in $T_{\text {rot }}$ and the mean energy of the upper rotational levels of the selected lines are given relative to the reference line set consisting of the first three $P_{1}$ - and $P_{2}$ branch lines. The plotted data points indicate the mean values and mean errors for the $\mathrm{OH}$ bands (4-1), (5-2), (6-2), and (7-4). Black circles and red diamonds show the results for the G98 and LG08 line parameters, respectively. The set of lines for each data point is indicated by an eight digit binary number. Selections are marked by the number 1 . The position of a digit corresponds to the wavelength of a $P$-branch line (see Fig. 2 and Table 1), i.e. the first and last digit refer to $P_{2}(1)$ and $P_{1}(4)$, respectively.

should be caused by uncertainties in the measurements and molecular data. The latter can explain the higher mean errors for the LG08 data and it might be a hint that these data are less accurate for $T_{\text {rot }}$ derivations than the G98 data (see also below).

The G98 $T_{\text {rot }}\left(v^{\prime}\right)$ results displayed in Fig. 6 indicate an increase in the temperature with $v^{\prime}$. $T_{\text {rot }}$ ranges from $188 \mathrm{~K}$ for $v^{\prime}=2$ to $202 \mathrm{~K}$ for $v^{\prime}=8$. Interestingly, the temperature increase is mainly limited to even $v^{\prime}$. Considering only odd $v^{\prime}, T_{\text {rot }}$ increases from $192 \mathrm{~K}$ for $v^{\prime}=3$ to $197 \mathrm{~K}$ for $v^{\prime}=9$, i.e. $\Delta T_{\text {rot }}$ is only $5 \mathrm{~K}$ compared to $14 \mathrm{~K}$ for even $v^{\prime}$. Consequently, the highest temperature is achieved for $v^{\prime}=8$, whereas $T_{\text {rot }}\left(v^{\prime}=9\right)$ is only the third highest. The differences in the $T_{\text {rot }}$ for odd and even $v^{\prime}$ are also clearly visible for fixed $\Delta v=v^{\prime}-v^{\prime \prime}$ and $v^{\prime} \geq 5$.

At least for $v^{\prime} \geq 6$, the $T_{\text {rot }}\left(v^{\prime}\right)$ gradient changes can also be observed for LG08 OH line parameters. Moreover, the temperature difference for even levels is larger than for odd ones ( 22 vs. $3 \mathrm{~K}$ ). For $v^{\prime}=7$, the second lowest temperature $(189 \mathrm{~K})$ was measured, which is $11 \mathrm{~K}$ lower than $T_{\text {rot }}\left(v^{\prime}=8\right)$. A difference of $8 \mathrm{~K}$ was found for $\mathrm{G} 98$ data. Therefore, we can assume that the striking $T_{\text {rot }}\left(v^{\prime}\right)$ pattern is not mainly caused by uncertainties in the $\mathrm{OH}$ line parameters. Nevertheless, the average LG08 $T_{\text {rot }}$ of all $v^{\prime}$ is about $4.4 \mathrm{~K}$ lower than the G98 value. In particular, $T_{\text {rot }}\left(v^{\prime}=2\right)$ derived from $\mathrm{OH}(2-0)$ is about $9 \mathrm{~K}$ lower.

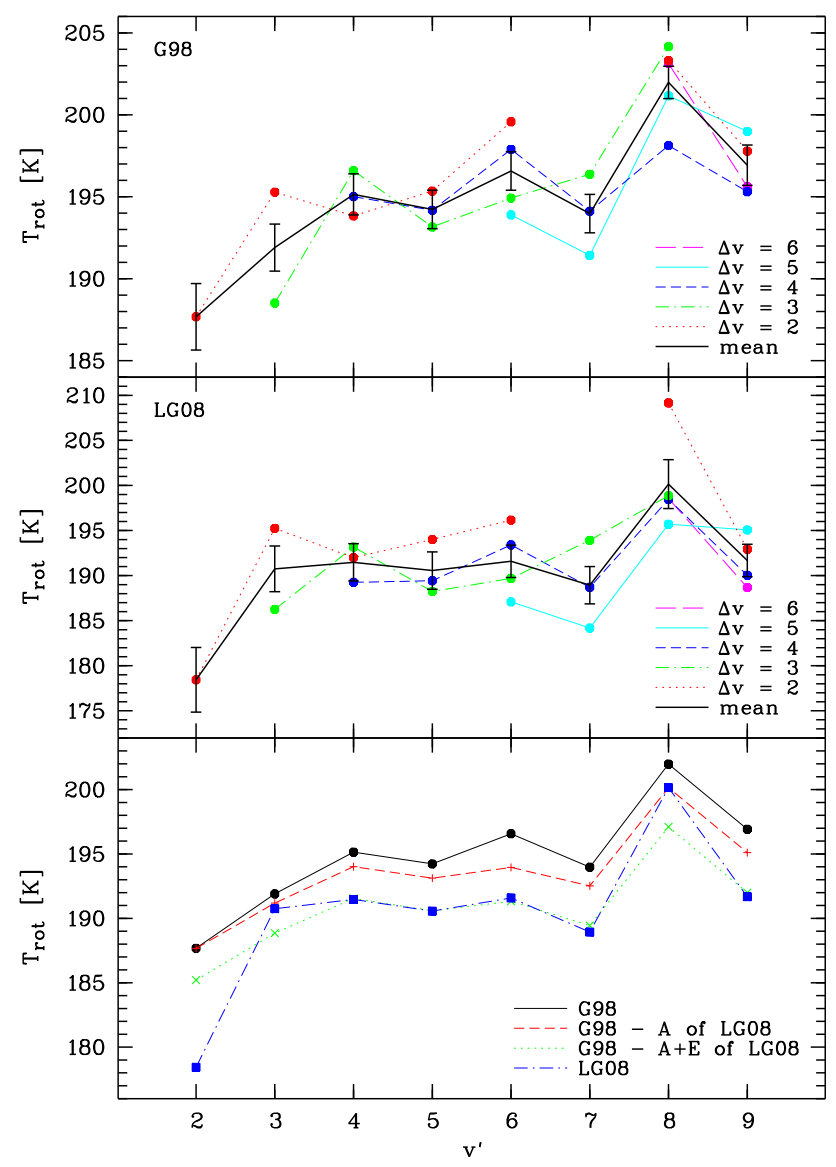

Figure 6. Rotational temperature $T_{\text {rot }}$ as a function of $v^{\prime}$. The filled circles in the upper and middle panel show the line-set-corrected $T_{\text {rot }}$ of the investigated $25 \mathrm{OH}$ bands for the G98 and LG08 line parameters, respectively. $T_{\text {rot }}$ for bands with the same vibrational level difference $\Delta v=v^{\prime}-v^{\prime \prime}$ are marked by different connecting lines (see legend). The averages of the $T_{\text {rot }}$ for bands with the same $v^{\prime}$ are connected by a black line, which also show the mean errors derived from the $T_{\text {rot }}$ variation for a given $v^{\prime}$ and the individual $T_{\text {rot }}$ measurement uncertainties. The lower panel exhibits the influence of the line parameters on $T_{\text {rot }}\left(v^{\prime}\right)$. Here, the results for the G98 data (black solid line), G98 data with the Einstein coefficients of LG08 (red dashed line), LG08 data with the atmospheric transmission correction and line integration wavelength ranges for G98 data (green dotted line), and the LG08 data (blue dash-dotted line) are shown (see also legend).

In the lower panel of Fig. 6, we show the reasons for these discrepancies. If $T_{\text {rot }}\left(v^{\prime}\right)$ is measured with the energy levels of G98 but the Einstein coefficients of LG08, the temperature decreases by $1.3 \mathrm{~K}$ on average for $v^{\prime}=2$ to 9 . For individual $v^{\prime}$, the shift is between $0.0 \mathrm{~K}\left(v^{\prime}=2\right)$ and $2.6 \mathrm{~K}$ $\left(v^{\prime}=6\right)$. If the LG08 energy levels are then used for the $T_{\text {rot }}$ derivation, the temperature further decreases by $2.7 \mathrm{~K}$ on average with only a small $v^{\prime}$ dependence (with individual shifts between 2.3 and $3.1 \mathrm{~K}$ ). Finally, the full LG08 results are achieved if the LG08 energy levels are also used for the 
line intensity measurements, i.e. for the wavelength range of the integration and the atmospheric transmission correction (see Sect. 3.2). The latter causes the large shift of $6.8 \mathrm{~K}$ for $T_{\text {rot }}\left(v^{\prime}=2\right)$, which is strongly affected by the water vapour absorption of $\mathrm{OH}(2-0)$ lines. Moreover, $T_{\mathrm{rot}}\left(v^{\prime}=8\right)$ is significantly changed by the very high LG08 $T_{\text {rot }}$ for $\mathrm{OH}(8-6)$, which is partly absorbed by $\mathrm{CO}_{2}$. The LG08 temperatures are less reliable than the G98 ones. The $\mathrm{OH}(2-0)$ and $\mathrm{OH}(8-6)$ line wavelengths from the LG08 data do not appear to be accurate enough for a good transmission correction. Although the line selection is based on G98 data (Sect. 3.1), which could cause a bias in favour of G98 data, the LG08 energy differences of the $\mathrm{OH}$ rotational levels seem to be less accurate than those of G98. This interpretation is supported by a higher variation of LG08 $T_{\text {rot }}$ for fixed $v^{\prime}$. Apart from the energies, the precision of the Einstein coefficients could also matter. In any case, the significant differences in the derived $T_{\text {rot }}$ of several $\mathrm{K}$ for these two up-to-date $\mathrm{OH}$ line parameter sets imply that absolute temperature measurements in the mesopause via this method are still very uncertain. To a lesser extent, this is also true for a comparison of $T_{\text {rot }}$ for different $v^{\prime}$, even if $\mathrm{OH}$ bands with significant atmospheric absorption are not considered.

The results presented in Fig. 6 are based on a sample of spectra taken at Cerro Paranal. Cosby and Slanger (2007) performed a similar analysis based on $16 \mathrm{OH}$ bands measured in 81 echelle spectra taken at Mauna Kea (see Sect. 1). Their derived $T_{\text {rot }}\left(v^{\prime}\right)$ pattern is in remarkable agreement with our results (see also Sect. 5.2.2). A similar trend could also be found by Cosby and Slanger (2007) for a mean UVVisual Echelle Spectrograph (UVES, Dekker et al., 2000) sky spectrum derived from several observations taken at Cerro Paranal in 2001 (Hanuschik, 2003). Although the data set is small, the striking $T_{\text {rot }}\left(v^{\prime}\right)$ pattern seems to be a general feature of $\mathrm{OH} T_{\text {rot }}$ measurements. Cosby and Slanger (2007) also preferably used G98 molecular data. Using the very different OH line parameters of Turnbull and Lowe (1989), their pattern did not change significantly except for a positive $T_{\text {rot }}$ shift. This confirms our result that systematic uncertainties in the line parameters do not appear to be responsible for the differences between odd and even $v^{\prime}$. It is more likely that this structure is caused by non-LTE effects related to $v^{\prime}$ dependent excitation and relaxation processes.

The most intense $\mathrm{OH}$ lines are those with $\Delta v=2$ (Mies, 1974; Rousselot et al., 2000; Khomich et al., 2008). Moreover, the nascent vibrational level distribution due to the hydrogen-ozone reaction (R1) is dominated by the high $v=$ 8 and 9 (Charters et al., 1971; Ohoyama et al., 1985; AdlerGolden, 1997). Therefore, peculiarities in the rotational level distributions of these $v$ could be transferred to lower $v$ causing the observed differences between odd and even $v^{\prime}$. Since the lowest $v$ can be populated by many different radiation processes, $T_{\text {rot }}$ differences for adjacent $v^{\prime}$ would be less significant, as it is observed. The scenario is more critical for the higher parts of the $\mathrm{OH}$ emission layer (e.g. Baker and Stair,
1988), since the lower $\mathrm{O}_{2}$ density reduces the frequency of quenching collisions, which tends to be a multiquantum process for at least high $v^{\prime}$ (McDade, 1991; Adler-Golden, 1997; $\mathrm{Xu}$ et al., 2012). The concentration of the other important quencher atomic oxygen $(\mathrm{O})$ increases with altitude in the mesopause region (e.g. Smith et al., 2010). However, the effect of O should mainly be a "sudden death" (McDade and Llewellyn, 1987) of the vibrationally excited OH preferentially by a chemical reaction with the products $\mathrm{H}$ and $\mathrm{O}_{2}$ (Adler-Golden, 1997; Varandas, 2004; Xu et al., 2012), i.e. the vibrational level distribution should not significantly change. Hence, the thermalisation of the remaining $\mathrm{OH}$ by collisions is less efficient at higher altitudes, i.e. signatures of the nascent level distribution can survive easier. Since the initial $T_{\text {rot }}$ of $v^{\prime}=8$ should be distinctly higher than for $v^{\prime}=9$ (e.g. 1230 vs. $760 \mathrm{~K}$ according to Llewellyn and Long, 1978), the measured $T_{\text {rot }}$ might still show higher values for $v^{\prime}=8$ due to the incomplete relaxation at high altitudes (see also Sect. 4.3). This could explain the observed $v^{\prime}$-specific differences. If this interpretation is correct, then especially $T_{\text {rot }}$ derived from $v^{\prime}=8$ should be taken with care. They appear to be several $\mathrm{K}$ too high. The second $T_{\text {rot }}$ peak is related to $v^{\prime}=6$, where $\mathrm{OH}(6-2)$ is frequently used (e.g. Beig et al., 2003, 2008). Odd and low $v^{\prime}$ seem to be less influenced by non-LTE effects, although non-negligible contributions are also likely.

For the odd levels, we find a $\Delta T_{\text {rot }} / \Delta v^{\prime}$ of about $0.74 \pm$ $0.21 \mathrm{~K}$ for G98 data. If non-LTE effects and line parameter uncertainties were negligible, this could be related to a real temperature gradient in the mesopause. A gradient is expected due to the dependence of the $\mathrm{OH}$ emission altitude on $v^{\prime}$, which is caused by the $\mathrm{O}_{2}$ and $\mathrm{O}$ density-related collisional quenching efficiency (McDade, 1991; Xu et al., 2012; von Savigny et al., 2012; von Savigny and Lednyts'kyy, 2013; Kowalewski et al., 2014). As indicated by OH emission profile measurements by rockets and satellites, the emission altitude can increase by about $0.5 \mathrm{~km}$ per vibrational level (von Savigny et al., 2012). This would result in a gradient of $+1.5 \mathrm{~K} \mathrm{~km}^{-1}$ at about $87 \mathrm{~km}$ for our Cerro Paranal sample mean. The real gradient could be an order of magnitude larger, since the emission layers for the different $v^{\prime}$ strongly overlap due to FWHM of about $8 \mathrm{~km}$ (Baker and Stair, 1988; von Savigny et al., 2012). Such steep mean temperature profiles appear to be unrealistic. Moreover, positive gradients would require a robust mesopause inversion layer (Meriwether and Gardner, 2000). However, lidar measurements at low latitudes suggest that the main mesopause temperature minimum is usually located at the top of or above the $\mathrm{OH}$ emission layer (Friedman and Chu, 2007; Clemesha et al., 2011), which implies a temperature decrease with altitude. Therefore, temperature gradients derived from $\mathrm{OH} T_{\text {rot }}$ appear to be mainly caused by non-LTE effects.

Our $\Delta T_{\text {rot }}$ can be compared with the $9.7 \pm 4.0 \mathrm{~K}$ found by Cosby and Slanger (2007) for their Mauna Kea $\left(19.8^{\circ} \mathrm{N}\right)$ sample. Since they used only $\mathrm{OH}(3-0)$ and $\mathrm{OH}(9-5)$ with 


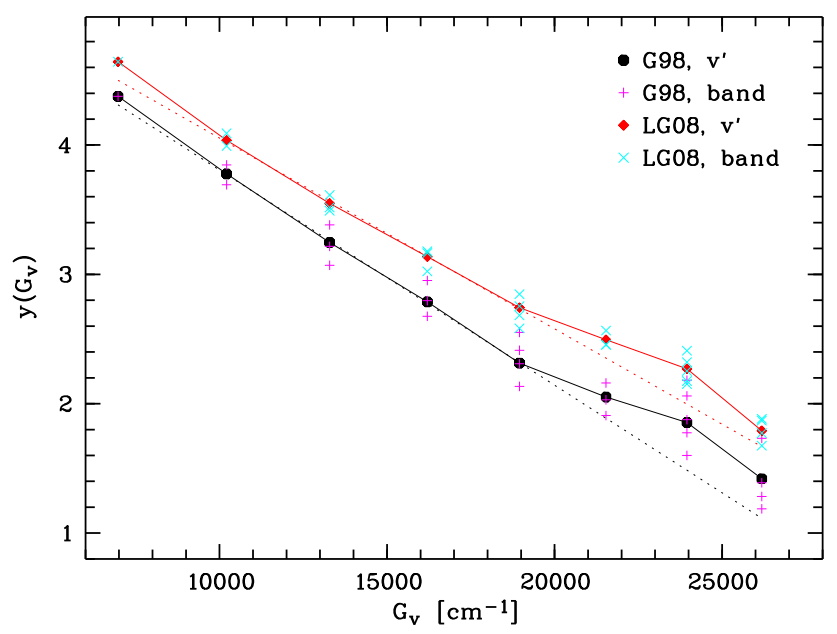

Figure 7. Derivation of $\mathrm{OH} T_{\mathrm{vib}}$ under the assumption of a Boltzmann distribution for the different $v^{\prime}$. The abscissa shows the energy of the lowest rotational level $G_{v}$ of the vibrational level $v^{\prime}$ in $\mathrm{cm}^{-1}$. The ordinate displays the natural logarithm of the population of a hyperfine-structure level of the lowest rotational level of $v^{\prime}$ relative to 1 Rayleigh second. The intensities of the $\mathrm{OH}$ lines originating from higher rotational levels were corrected by means of the $v^{\prime}$-related $T_{\text {rot }}$ (see Fig. 6) to correspond to the rotational ground state of $X^{2} \Pi_{3 / 2}$. The "+" and " $\times$ " symbols show the mean values of $y\left(G_{v}\right)$ for each $\mathrm{OH}$ band for the G98 and LG08 line parameters, respectively. The averaged $y\left(G_{v}\right)$ from all lines with fixed $v^{\prime}$ are displayed by filled black circles and red diamonds and are connected by solid lines. The dotted lines represent linear fits to the averages for $v^{\prime}=3$ to 6 .

different line sets for this value, we estimated a temperature difference with the same lines. In view of the possible variability on different timescales (see Sect. 5), our result of $6.9 \pm 3.9 \mathrm{~K}$ is in good agreement.

\subsection{Vibrational level populations and temperatures}

As described in Sect. 3.4, we can compare the populations of different vibrational levels by extrapolating the $y$ values of the measured lines to the corresponding ground states $G_{v}$. This requires the derived $T_{\text {rot }}$ and the energy differences $E^{\prime}-G_{v}$. Figure 7 shows the resulting population-energy plot for the band- and $v^{\prime}$-specific averages derived from the sample mean intensities. The band-specific averages are highly reliable, since the typical scatter in $y$ resulting from the measured lines of a band is only 0.03 . The scatter is significantly larger if $\mathrm{OH}$ bands with the same $v^{\prime}$ are compared, especially for high $v^{\prime}$. The $y$ difference for $\mathrm{OH}(8-6)$ and $\mathrm{OH}(8-2)$ is 0.58 with the G98 line parameters. For $\mathrm{OH}(9-7)$ and $\mathrm{OH}(9-$ 3 ), the difference is 0.55 , which corresponds to a population ratio of about 1.7. The corresponding numbers for LG08 data are 0.02 and -0.10 . Here, the maximum differences are 0.26 and 0.21 for $\mathrm{OH}(8-4)$ minus $\mathrm{OH}(8-2)$ and $\mathrm{OH}(9-4)$ minus $\mathrm{OH}(9-7)$, respectively. As the deviations (and also the abso- lute $y$ values) are very different for G98 and LG08 data, they are most likely caused by uncertainties in the molecular line parameters. In contrast to the results for $T_{\text {rot }}$ (Sect. 4.2), the LG08 data appear to be more reliable in this context due to the smaller differences. In view of the systematic deviations, any $v^{\prime}$ population comparison has to be taken with care. The resulting values depend on the considered $\mathrm{OH}$ bands for each $v^{\prime}$. For example, the missing $\mathrm{OH}(9-7)$ band (see Sect. 3.1) causes a decrease of $y\left(v^{\prime}=9\right)$ for G98 data. Therefore, our line selection inevitably affects the vibrational level populations and temperatures, which we discuss in the following.

The average $y\left(v^{\prime}\right)$ derived from all measured lines show a clear decrease with increasing $v^{\prime}$. However, this decrease cannot be explained by a single Boltzmann distribution, as it is indicated by the dotted line for each line parameter set. The $y\left(v^{\prime}\right)$ fits were derived with $v^{\prime}$ from 3 to 6 , which indicate a good linear relation. The deviations for $v^{\prime}=2$ might be explained by uncertainties related to the atmospheric absorption correction for the $\mathrm{OH}(2-0)$ band, where the G98 results are more reliable (see Sect. 4.3). The overpopulations for $v^{\prime}=7$ to 9 relative to the fit are highly significant, although the amounts depend on the selected lines and bands. We find a maximum deviation for $v^{\prime}=8$, i.e. 0.37 (overpopulation of $45 \%)$ for G98 and 0.27 (31\%) for LG08 data. The increased population of $v^{\prime}=8$ has been observed before based on a smaller number of $\mathrm{OH}$ bands (Takahashi and Batista, 1981; Cosby and Slanger, 2007) and was also modelled by Adler-Golden (1997). The nascent vibrational level distribution preferring high $v$ (Llewellyn and Long, 1978; McDade and Llewellyn, 1987) appears to partly survive the radiative cascades and vibrational relaxation via collisions (McDade, 1991; Adler-Golden, 1997; Xu et al., 2012; von Savigny et al., 2012). Therefore, the found relative overpopulation for the highest $v^{\prime}$ could significantly contribute to the $T_{\text {rot }}\left(v^{\prime}\right)$ gradients discussed in Sect. 4.2.

The observed population pattern implies that $T_{\mathrm{vib}}$ (Sect. 3.4) strongly depend on the selected $v^{\prime}$ for the regression analysis. For all covered $v^{\prime}$ from 2 to 9 , we derive $10170 \pm 200 \mathrm{~K}$ for G98 data and $10760 \pm 140 \mathrm{~K}$ for LG08 data by taking individual $y$ for all measured $\mathrm{OH}$ lines. When only the vibrational levels 3 to 6 are considered, as shown in Fig. 7, we obtain $8660 \pm 240 \mathrm{~K}$ and $9830 \pm 210 \mathrm{~K}$, respectively. Consequently, the high- $v$ population bump changes $T_{\text {vib }}$ by about 1500 or $900 \mathrm{~K}$ depending on the $\mathrm{OH}$ line parameters. In any case, the resulting $T_{\mathrm{vib}}$ are close to those found by other studies (see Khomich et al., 2008).

\section{Variability}

The atmosphere is highly dynamic. By investigating $\mathrm{OH}$ rotational temperatures and vibrational level populations as a function of time and band, we can further constrain the origin of their $v^{\prime}$-dependent values. We will start with a discussion of a small time series that covers a whole night (Sect. 5.1). Then, we will describe nocturnal (Sect. 5.2.1) 
and seasonal variations (Sect. 5.2.2) for the whole sample of 343 spectra (Sect. 2.2). Finally, we will discuss possible correlations of the quantities that were derived from the individual observations (Sect. 5.2.3). The results in this section are mainly based on G98 line parameters due to their higher $T_{\text {rot }}$ reliability (see Sect. 4.2).

\subsection{An example time series}

Our sample of X-shooter spectra includes 17 observations that were taken within $11 \mathrm{~h}$ in the night of 29/30 July 2011. The exposure times were $35 \mathrm{~min}$ ( $17.5 \mathrm{~min}$ for the first and last exposures). Since only a single astronomical target was observed, zenith distance and azimuth angle changed as a function of time. The zenith distance ranged from 23 to $65^{\circ}$, with the minimum near midnight. This small but high-quality sample allows us to evaluate how temperatures derived from different $\mathrm{OH}$ bands are dynamically connected.

The upper panel of Fig. 8 shows the $T_{\text {rot }}$ for the reference line set (see Sect. 3.3) and G98 data derived from $15 \mathrm{OH}$ bands. For each $v^{\prime}$, the results for the two most reliable bands are displayed. An exception is $v^{\prime}=2$, where only $\mathrm{OH}(2-0)$ is available. The $T_{\text {rot }}$ are given as a function of the local time, i.e. UT corrected for the longitude of Cerro Paranal, and it is given for the middle of the exposure. The measured $T_{\text {rot }}$ distribution is in good agreement with the results for the full sample mean (Sect. 4.2). While the highest temperatures tend to be found for $v^{\prime}=8(197 \mathrm{~K}$ on average for $\mathrm{OH}(8-5))$, the lowest $T_{\text {rot }}$ of the night are related to $\mathrm{OH}(2-0)(177 \mathrm{~K}$ on average). Moreover, $\mathrm{OH}$ bands with the same $v^{\prime}$ behave similarly. This can be shown by calculating Pearson's correlation coefficient $r$, which turned out to be 0.993 on average for the plotted band combinations. This is a strong argument for the interpretation that $T_{\text {rot }}$ of $\mathrm{OH}$ bands with the same $v^{\prime}$ mostly differ due to measurement uncertainties and inaccurate line parameters, i.e. Einstein coefficients and level energies.

A comparison of $\mathrm{OH}$ bands with different $v^{\prime}$ implies a variability pattern that depends on $v^{\prime}$. We found a monotonically decreasing correlation coefficient with increasing $\Delta v^{\prime}$. For the plotted bands and e.g. $\Delta v^{\prime}=1,4$, and 7 , the average $r$ is $0.987,0.917$, and 0.797 , respectively. First, these increasing discrepancies are caused by a shift of the nocturnal temperature minimum towards later times for higher $v^{\prime}$. The maximum time difference is about $2 \mathrm{~h}$. Second, the temperature variations in the investigated night were larger for lower $v^{\prime}$. They ranged from $32 \mathrm{~K}$ for $\mathrm{OH}(9-4)$ to $46 \mathrm{~K}$ for $\mathrm{OH}(2-$ $0)$. These large $T_{\text {rot }}$ variations took place in the first half of the night, since the first spectrum always showed the maximum temperature. The $T_{\text {rot }}$ increase in the second half of the night does not indicate a significant $v^{\prime}$ dependence. Finally, the $T_{\text {rot }}$ for the different plotted bands are remarkably similar at the beginning of the night. The spread was only about $11 \mathrm{~K}$. It rapidly increased before midnight, when the $T_{\text {rot }}$ minima were reached, and stayed large until the end of the night $(31 \mathrm{~K})$ with some variations.

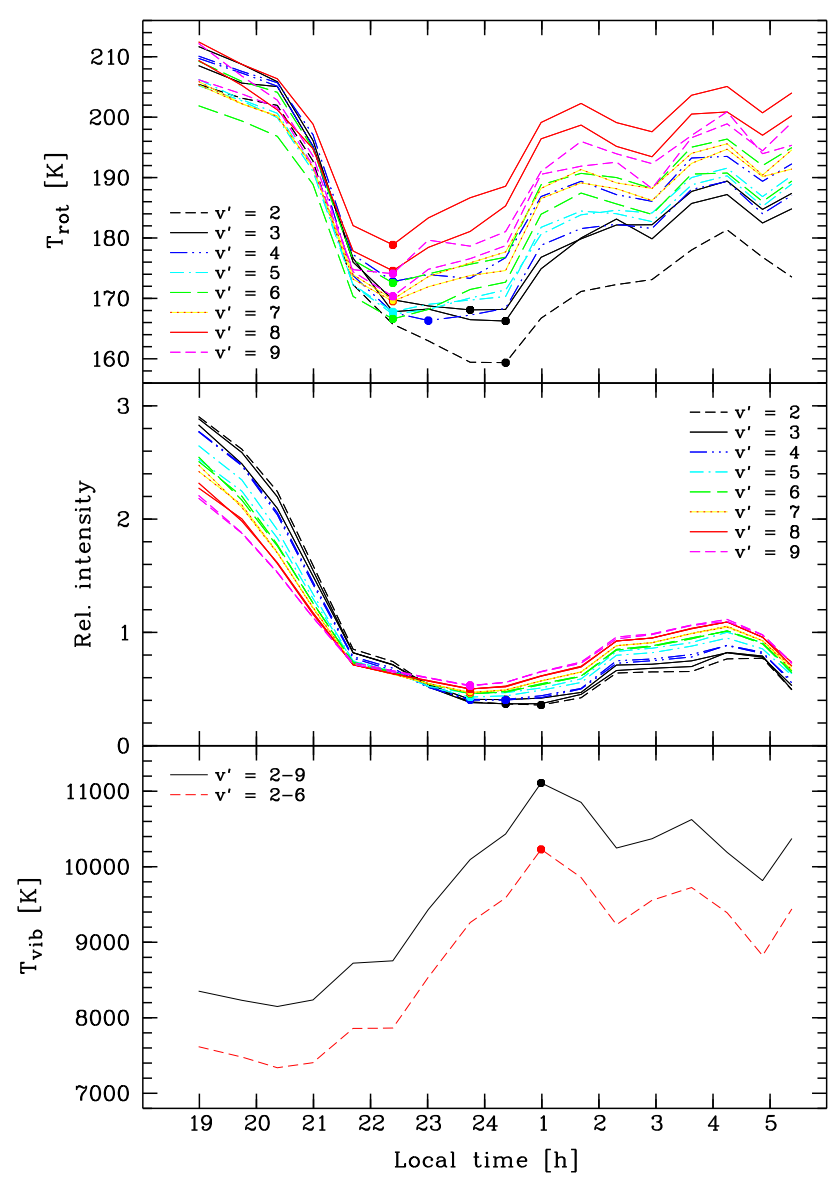

Figure 8. $T_{\text {rot }}$, relative intensity, and $T_{\text {vib }}$ as a function of local time (UT corrected for geographical longitude) for a time series of 17 X-shooter observations taken during the night 29/30 July 2011. All data points are based on the G98 line parameters. The upper panel shows the line-set-corrected $T_{\text {rot }}$ for the $\mathrm{OH}$ bands (2-0), (3-0), (31), (4-1), (4-2), (5-2), (5-3), (6-2), (6-3), (7-3), (7-4), (8-3), (8-5), (9-4), and (9-5). Bands with the same $v^{\prime}$ are marked by lines of the same type and colour (see legend). The observation of each band with the lowest $T_{\text {rot }}$ is indicated by a filled circle. The middle panel shows the intensity of the same $\mathrm{OH}$ bands as listed above relative to the average for the 17 observations of the sample. The lowest intensity of each band is marked by a filled circle. The lower panel displays $T_{\mathrm{vib}}$ derived from the lines of the bands listed above for two different $v^{\prime}$ sets ( 2 to 9 and 2 to 6 , see legend). The observations indicating the highest $T_{\mathrm{vib}}$ are marked by filled circles.

A strong $v^{\prime}$ dependence of the variability can also be found for the band intensity, which traces the vibrational level population and is shown in the middle panel of Fig. 8. The relative intensities were derived from the summed line intensities for each band and normalised to the mean of the 17 observations. All $\mathrm{OH}$ bands indicate a steep intensity decrease at the beginning, a minimum in the middle of the night, a weak intensity increase in the second half, and a second minimum at the end of the night. While bands with the same $v^{\prime}$ show curves which are almost identical, there is a continu- 
ous change in the variability pattern from $v^{\prime}=2$ to 9 . The intensity decrease at the beginning becomes weaker (factors from 8.1 to 4.1 ) and the minimum is earlier for higher $v^{\prime}$. This trend is very similar to the results for $T_{\text {rot }}$, which show an average minimum that was about 70 min earlier than the one for the intensity. The time difference appears to decrease for lower $v^{\prime}$.

Our data clearly show a changing $T_{\text {rot }}$ and intensity variability from $v^{\prime}=2$ to 9 . The general features of these variations were observed before based on studies with a smaller number of bands, lower-quality data, and/or smaller data sets (Takahashi and Batista, 1981; Cosby and Slanger, 2007; Perminov et al., 2007). In particular, Takahashi and Batista (1981) observed five $\mathrm{OH}$ bands in 64 nights at Cachoeira Paulista in Brazil (see Sect. 1), which is at a similar latitude $\left(22.7^{\circ} \mathrm{S}\right)$ as Cerro Paranal $\left(24.6^{\circ} \mathrm{S}\right)$. Their data resulted in minimum times for $T_{\text {rot }}$ and intensities that were similar to ours. Moreover, they found that the nocturnal variation of the intensity of $\mathrm{OH}(5-1)$ was delayed by about $15 \mathrm{~min}$ compared to $\mathrm{OH}(9-4)$. Although our time resolution is lower, this shift seems to be consistent with the delays that we derive from larger $\Delta v^{\prime}$. The found $v^{\prime}$-related variations can only be explained if the altitude of the $\mathrm{OH}$ emission depends on $v^{\prime}$, which is consistent with results of observations based on rockets and satellites (Lopez-Moreno et al., 1987; Baker and Stair, 1988; Xu et al., 2012; von Savigny et al., 2012). Our example data suggest that the emission layer altitude was a monotonic function of $v^{\prime}$ for $v^{\prime}=2$ to 9 . As the layer profile measurements and corresponding models imply that higher $v^{\prime}$ tend to be at higher altitudes (Adler-Golden, 1997; Xu et al., 2012; von Savigny et al., 2012), our measurements indicate a strong positive temperature gradient with altitude after the $T_{\text {rot }}$ minima, even if the $v^{\prime}=8$ bands are neglected due to major non-LTE contributions (see Sect. 4.2). In contrast, the gradient was close to zero at the beginning of the night. Apart from real changes in the mesopause temperature profile, this can be caused by changes in the altitude and spread of the emission layers for the different $v^{\prime}$, which would modify the $v^{\prime}$-related effective temperature. The $\mathrm{OH}$ emission distribution is affected by nocturnal altitude-dependent concentration variations of the reactants $\mathrm{O}_{3}$ and $\mathrm{H}$ as well as the main quenchers $\mathrm{O}_{2}$ and $\mathrm{O}$ (e.g. Kowalewski et al., 2014). In particular, the vertical transport and concentration change of $\mathrm{O}$ is critical, as it also influences the $\mathrm{O}_{3}$ concentration (e.g. Marsh et al., 2006). Since such processes can lead to $v^{\prime}$-dependent changes in the $\mathrm{OH}$ collisional quenching efficiency, we can expect $v^{\prime}$-related variations in the non-LTE $T_{\text {rot }}$ contributions. If this mainly causes the observed increasing $T_{\text {rot }}$ differences, then a rise of (at least parts of) the $\mathrm{OH}$ emission layer would be required to reduce the vibrational relaxation by $\mathrm{O}_{2}$ collisions. It would be consistent with the observed intensity evolution, since there appears to be an inverse relation between the $\mathrm{OH}$ integrated emission rate and the emission peak altitude (Yee et al., 1997; Melo et al., 1999; Liu and Shepherd, 2006). Figure 8 also indicates that struc- tures in the variability pattern for $T_{\text {rot }}$ and intensity appear to move from high emission layers (high $v^{\prime}$ ) to low layers (low $v^{\prime}$ ). For example, the marked $T_{\text {rot }}$ and intensity minima are later for lower $v^{\prime}$. This behaviour points to thermal tides, which show these phase shifts by propagation from the lower atmosphere forced by solar heating (e.g. A. K. Smith, 2012). For low latitudes, the solar $(1,1)$ migrating diurnal tide should dominate (Vincent et al., 1988; Burrage et al., 1995; Marsh et al., 2006). The $T_{\text {rot }}$ variation for $v^{\prime}=2$, which is least influenced by non-LTE effects, supports the assumption of a dominating $24 \mathrm{~h}$ period. Our data also suggest some contribution from shorter period variations like those with a period of 2 to $3 \mathrm{~h}$ in the second half of the night, which could be related to long-period gravity waves (e.g. Taylor et al., 1997; Khomich et al., 2008). Note that the true period probably deviates due to the moving telescope. Finally, it should be noted that the nocturnal intensity evolution, especially the high values at the beginning of the night, is strongly affected by the $\mathrm{OH}$ photochemistry, i.e. the changing availability of the reactants ozone and hydrogen (e.g. Yee et al., 1997).

The lower panel of Fig. 8 shows the variation of two different $T_{\text {vib }}$ related to the $v^{\prime}$ ranges 2 to 9 and 2 to 6 , respectively. The latter excludes those vibrational levels which indicate an overpopulation compared to the lower ones (see Sect. 4.3). Like for $T_{\text {rot }}$, the G98 line parameters were used for the calculations. The general structure of the variations for both quantities is a relatively low $T_{\text {vib }}$ at the beginning, followed by an increase with a maximum after midnight. The rest of the night, the $T_{\mathrm{vib}}$ results indicate some fluctuations with a weak trend towards lower temperatures. $T_{\mathrm{vib}}(2-9)$ is higher than $T_{\mathrm{vib}}(2-6)$ due to the additional population at high $v^{\prime}$. However, the difference between both $T_{\text {vib }}$ is relatively stable. The mean value is $870 \mathrm{~K}$ and the scatter is only $80 \mathrm{~K}$. This points to similar relative changes for the basic population distribution measured by $T_{\mathrm{vib}}(2-6)$ and the additional population specific for high $v^{\prime}$. With a minimum difference of $740 \mathrm{~K}$ for the first data point, there is only a weak trend towards a lower impact of the high- $v^{\prime}$ overpopulation at the beginning of the night. The vibrational temperature is anticorrelated with the intensity. For $T_{\mathrm{vib}}(2-9)$ in combination with the intensity curves of the different bands, we obtain $r$ between -0.60 and -0.80 . Consequently, high $\mathrm{OH}$ vibrational populations are related to small high- $v /$ low- $v$ population ratios and vice versa. This relation and the trend of increasing $T_{\text {vib }}$ in the first half of the night are in agreement with the assumptions reported by Cosby and Slanger (2007).

\subsection{Variations in the full sample}

The discussion of the example time series in Sect. 5.1 has already been valuable in terms of results on the $v^{\prime}$ dependence of variations in $T_{\text {rot }}$, intensities, and $T_{\text {vib. }}$. However, these data only cover one night. In order to evaluate the generality of these results (at least for Cerro Paranal), we investigated the full sample of $343 \mathrm{X}$-shooter spectra (see Sect. 2.2). 


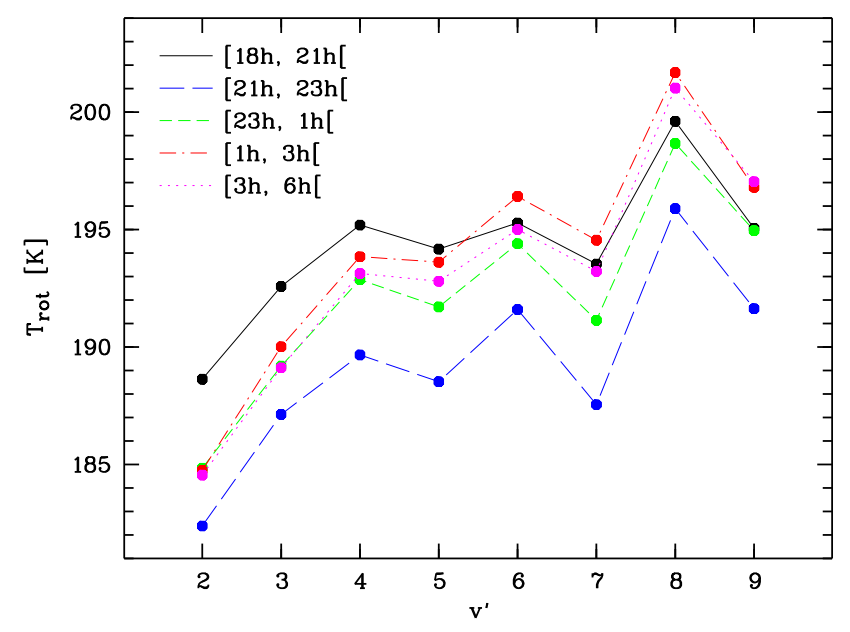

Figure 9. $T_{\text {rot }}\left(v^{\prime}\right)$ as a function of $v^{\prime}$ for different local time periods. Data points for the same period are connected by lines of a fixed type and colour (see legend). All data points are based on the G98 line parameters. The errors of the plotted mean values are of the order of $1 \mathrm{~K}$

\subsubsection{Nocturnal variations}

First, we calculated mean $T_{\text {rot }}\left(v^{\prime}\right)$ from the individual observations in different time bins to analyse the nocturnal variations. The sample mean values are expected to be representative of the true annual averages due to the well-balanced time and day distribution in our sample (see Sect. 2.2). The individual $T_{\text {rot }}\left(v^{\prime}\right)$ were derived from the average of the bandrelated $T_{\text {rot }}$ with the same $v^{\prime}$. In contrast to Sect. 4.2, we neglected the faintest band $\mathrm{OH}(8-2)$ for a higher reliability of the $v^{\prime}=8$ results (see Sect. 3.3). It also should be noted that the sample-averaged $T_{\text {rot }}$ derived from intensities of single spectra slightly differ from the $T_{\text {rot }}$ based on the sample mean intensities discussed in Sect. 4.2. For all spectra and G98 line parameters, we obtain differences between $-1.7\left(v^{\prime}=9\right)$ and $-2.6 \mathrm{~K}\left(v^{\prime}=2\right)$. Despite the discrepancies, we applied both methods because of either the high robustness for studying individual lines and bands or the high flexibility for analysing the variability.

Figure 9 shows the resulting mean $T_{\text {rot }}\left(v^{\prime}\right)$ for five different time bins with a size of 2 to $3 \mathrm{~h}$ and 62 to 75 spectra. The data are based on the G98 parameters. The curves of all the bins reveal the characteristic pattern discussed in Sect. 4.2. The $T_{\text {rot }}$ of even $v^{\prime}$ tend to be higher than the ones of odd $v^{\prime}$. The temperatures of $v^{\prime}=2$ and 8 are always the lowest and highest ones, respectively. The persistence of this structure supports our interpretation that it is caused by different degrees of thermalisation related to the nascent level distribution, the strength of $\Delta v=2$ transitions, and the vibrational relaxation efficiency by collisions, which is lower at higher altitudes. Interestingly, the $T_{\text {rot }}\left(v^{\prime}\right)$ pattern appears to be more pronounced for those bins with the lowest temperatures. In particular, the strength of the $v^{\prime}=6$ peak changes.

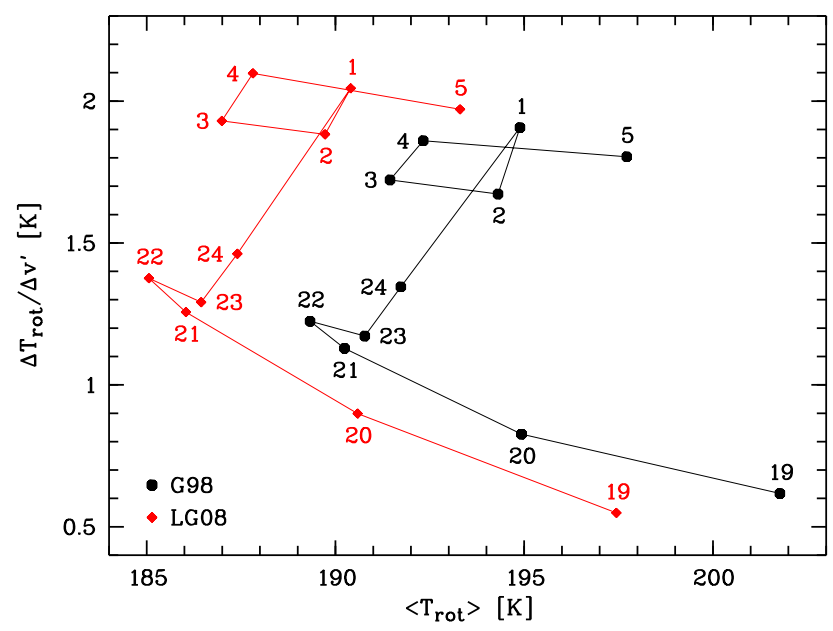

Figure 10. Change of $T_{\text {rot }}$ with $v^{\prime} \Delta T_{\text {rot }} / \Delta v^{\prime}$ (slope of regression line) as a function of the average $\left\langle T_{\text {rot }}\right\rangle$ of all $v^{\prime}$ for local time bins centred on the hour (see labels). The plot is based on the $T_{\text {rot }}\left(v^{\prime}\right)$ of the individual spectra. Results are shown for the G98 (black filled circles) and LG08 line parameters (red filled diamonds), respectively.

The difference between $T_{\text {rot }}$ for $v^{\prime}=6$ and 7 varies between 1.8 and $4.1 \mathrm{~K}$. On the other hand, the range of $T_{\text {rot }}$ differences between $v^{\prime}=8$ and 9 of 3.7 to $4.9 \mathrm{~K}$ is only half as large $(1.2$ vs. $2.3 \mathrm{~K})$. An increasing discrepancy between adjacent $v^{\prime}$ could point to a rise of the emission layer, as this would reduce the density and hence weaken the vibrational relaxation by $\mathrm{O}_{2}$ collisions. Non-LTE effects would then be stronger.

The overall nocturnal $T_{\text {rot }}$ evolution in Fig. 9 indicates a significant decrease at the beginning of the night and a slower increase afterwards. This is also shown by Fig. 10, which exhibits the average $T_{\text {rot }}$ for all $v^{\prime}$ and $1 \mathrm{~h}$ bins centred on the hour. The minimum is at about 22:00 LT. Since this is the result regardless of the G98 and LG08 line parameters, their choice does not seem to be critical in terms of temporal variations. On the other hand, there is a typical difference in the absolute temperature of about $4 \mathrm{~K}$, which illustrates that absolute values are still very uncertain. Figures 9 and 10 also reveal a $v^{\prime}$ dependence of $T_{\text {rot }}$ depending on time. The slope $\Delta T_{\text {rot }} / \Delta v^{\prime}$ derived from all $v^{\prime}$ tends to increase through the entire night, irrespective of the molecular data. For G98 data, it ranges between 0.6 (19:00 LT) and $1.8 \mathrm{~K}(05: 00 \mathrm{LT})$ when all $v^{\prime}$ from 2 to 9 are involved in the calculation. As the nonLTE contributions seem to differ for odd and even $v^{\prime}$, we also calculated quantities for only these subsets. While the timeaveraged absolute $T_{\text {rot }}$ differ by about $0.7 \mathrm{~K}$ for these subsets and the average $\Delta T_{\text {rot }} / \Delta v^{\prime}$ is $0.8 \mathrm{~K}$ for odd $v^{\prime}$ and $2.2 \mathrm{~K}$ for even $v^{\prime}$ (as derived from the $1 \mathrm{~h}$ bins), the nocturnal trends are very similar with an uncertainty of a few tenth K. A reason for a small difference in the trends could be the $T_{\text {rot }}\left(v^{\prime}\right)$ pattern change for intermediate $v^{\prime}$ discussed above. 


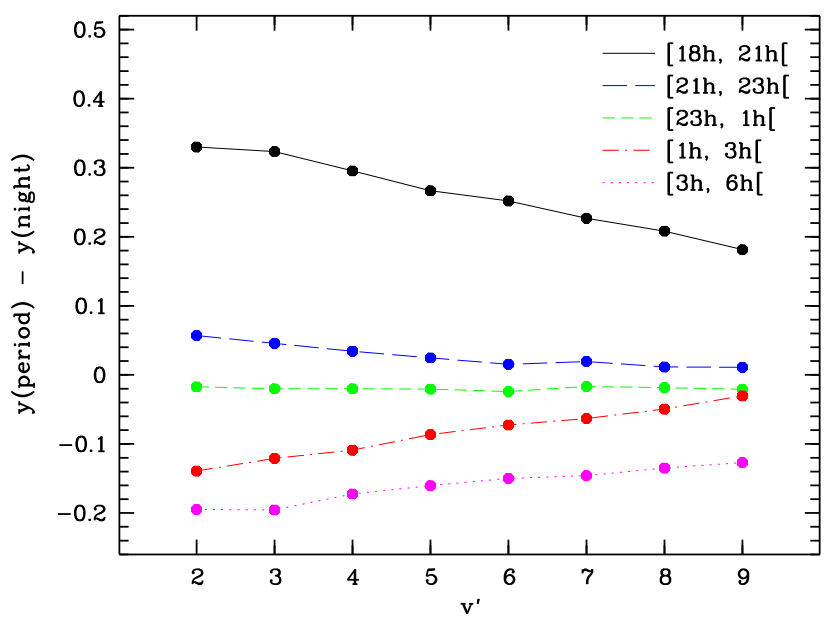

Figure 11. Vibrational population ratio relative to the sample mean as a function of $v^{\prime}$ for different local time periods. Data points for the same period are connected by lines of a fixed type and colour (see legend). All data points are based on the G98 line parameters. The $y$ values were derived from the intensities of all measured lines. The errors of the plotted mean values are of the order of 0.04 .

The nocturnal $T_{\text {rot }}$ variations for the full sample show the same general features as those for our example night discussed in Sect. 5.1, which implies that this night was representative. There is also a good agreement with other measurements at a similar latitude. Takahashi et al. (1998) studied OH(9-4) observations at Cachoeira Paulista, Brazil $\left(22.7^{\circ} \mathrm{S}\right)$ and Gelinas et al. (2008) analysed measurements from $\mathrm{OH}(6-2)$ at Alice Springs, Australia $\left(23.7^{\circ} \mathrm{S}\right)$. Interestingly, the average nocturnal $T_{\text {rot }}$ maximum appeared to be close to the end of the night, which is consistent with our results for high $v^{\prime}$. For low $v^{\prime}$, our results suggest that their maximum would have been more likely at the beginning of the night. This illustrates the importance of the $v^{\prime}$ of the observed band for the interpretation of the dynamics.

In the same way as $T_{\text {rot }}$ (Fig. 9), we also analysed the vibrational level populations. Figure 11 shows the $v^{\prime}$ populations for the five different night bins relative to the nocturnal mean in logarithmic units for the G98 data. The figure reveals a general decrease of the populations in the course of the night. The population at the beginning is on average about $50 \%$ higher than at the end. In comparison, the intensity variations in our example night indicate a distinctly stronger drop but only until midnight (see Sect. 5.1). The subsequent increase cannot be found in the averaged data. The nocturnal intensity variations investigated by Takahashi et al. (1998) and Gelinas et al. (2008) for different months are also more complex. However, our simple averaging over several years (which were also different from those of the other studies) could explain the discrepancies. A more detailed study requires a larger data set. Nevertheless, our data are well suited to study the $v^{\prime}$ dependence of the level population. Here, we clearly see a faster depopulation of the lower

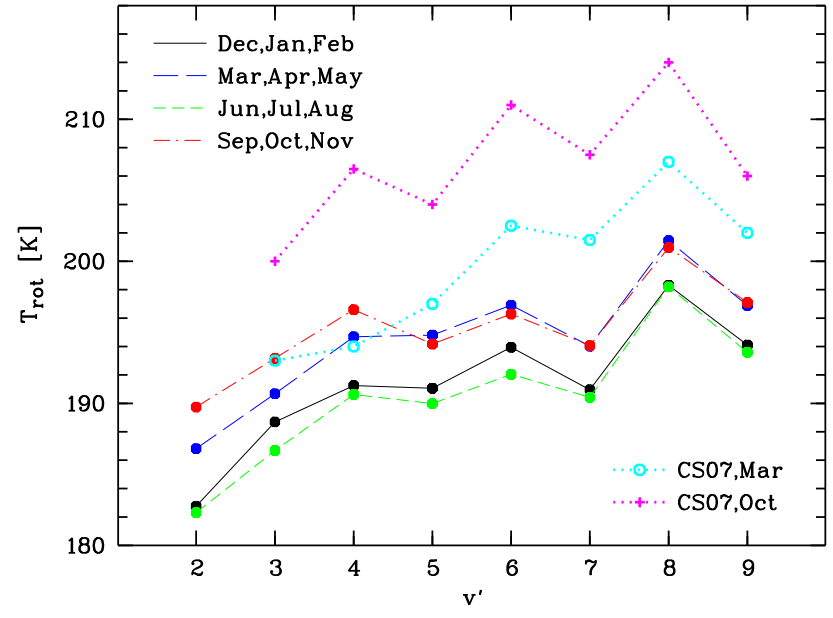

Figure 12. Rotational temperature $T_{\text {rot }}\left(v^{\prime}\right)$ as a function of $v^{\prime}$ for different meteorological seasons. The plot details are similar to those of Fig. 9. In addition, we show the Mauna Kea $\left(19.8^{\circ} \mathrm{N}\right)$ mean $T_{\text {rot }}\left(v^{\prime}\right)$ for March and October 2000 by Cosby and Slanger (2007).

$v^{\prime}$, in accordance with the discussion in Cosby and Slanger (2007). Since the plotted curves are close to straight lines, the high $v^{\prime}$ characterised by an overpopulation relative to the lower $v^{\prime}$ (see Sect. 4.3) do not appear to show a special behaviour. The $v^{\prime}$-dependent changes in the level populations of all $v^{\prime}$ can be well described by $T_{\text {vib }}(2-9)$ differences. The resulting $\Delta T_{\mathrm{vib}}(2-9)$ for the fourth (maximum of $10030 \mathrm{~K}$ ) and the first bin (minimum of $9180 \mathrm{~K}$ ) is $860 \mathrm{~K}$. The $T_{\mathrm{vib}}(2-9)$ of the full sample amounts to $9660 \mathrm{~K}$. Note that these values differ from those given in Sect. 4.3, since they were derived from the mean $y$ values in Fig. 11 and not the $y$ values of the individual $\mathrm{OH}$ lines.

\subsubsection{Seasonal variations}

We also investigated seasonal variations. Due to the small sample size, we divided the year only into the four meteorological seasons. Figure 12 shows the corresponding results for $T_{\text {rot }}\left(v^{\prime}\right)$ based on 68 (autumn) to 117 spectra (summer). There are two pairs of curves. Autumn and spring are very similar, except for possible slight deviations at low $v^{\prime}$. Moreover, the summer and winter averages indicate a good agreement. This suggests a dominance of a semi-annual oscillation with a low amplitude, which is somewhat expected due to the low latitude of Cerro Paranal. A similar pattern could also be found by Takahashi et al. (1995) with $\mathrm{OH}(9-4)$ for Cachoeira Paulista and Gelinas et al. (2008) with $\mathrm{OH}(6-2)$ for Alice Springs. For both data sets, the amplitude of the semiannual oscillation was about $4 \mathrm{~K}$, whereas the amplitude of the annual oscillation was almost zero. Our $\Delta T_{\text {rot }} / \Delta v^{\prime}$ derived from all $v^{\prime}$ are very similar from winter to summer with about $1.6 \mathrm{~K}$. A lower slope of $1.0 \mathrm{~K}$ was found in spring, i.e. for September to November. The March and October 2000 data of Cosby and Slanger (2007) for Mauna Kea $\left(19.8^{\circ} \mathrm{N}\right)$ 
Table 2. Correlation coefficients $r$ for different quantities derived from the individual observations by means of G98 and LG08 line parameters.

\begin{tabular}{llcc}
\hline Quantity A & Quantity B & $r$ (G98) & $r$ (LG08) \\
\hline$\left\langle T_{\operatorname{rot}}\left(v^{\prime}\right)\right\rangle$ & $\Delta T_{\text {rot }} / \Delta v^{\prime}$ & -0.063 & +0.025 \\
$\left\langle T_{\operatorname{rot}}\left(v^{\prime}\right)\right\rangle$ & $n /\langle n\rangle\left(v^{\prime}=5\right)$ & +0.601 & +0.607 \\
$\left\langle T_{\operatorname{rot}}\left(v^{\prime}\right)\right\rangle$ & $T_{\text {vib }}(2-9)$ & +0.235 & +0.245 \\
$\Delta T_{\operatorname{rot}} / \Delta v^{\prime}$ & $n /\langle n\rangle\left(v^{\prime}=5\right)$ & -0.565 & -0.448 \\
$\Delta T_{\operatorname{rot}} / \Delta v^{\prime}$ & $T_{\text {vib }}(2-9)$ & +0.589 & +0.431 \\
$n /\langle n\rangle\left(v^{\prime}=5\right)$ & $T_{\mathrm{vib}}(2-9)$ & -0.426 & -0.375 \\
\hline
\end{tabular}

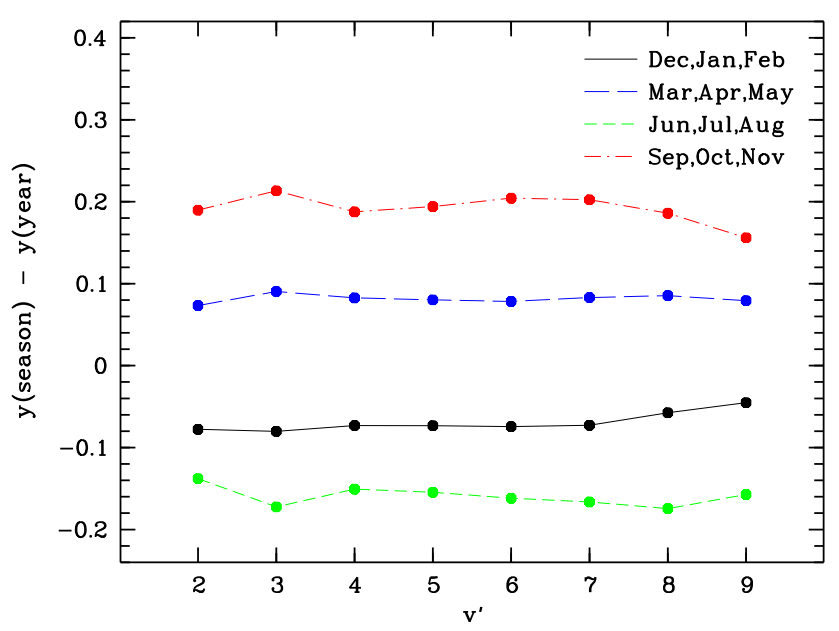

Figure 13. Vibrational population ratio relative to the sample mean as a function of $v^{\prime}$ for different meteorological seasons. The plot details are similar to those of Fig. 11.

indicate a steeper slope for March (see Fig. 12). We estimate $2.1 \mathrm{~K}$ (March) and $1.3 \mathrm{~K}$ (October) for $v^{\prime}=3$ to 9 . Hence, Mauna Kea and Cerro Paranal data taken in a similar period of the year appear to agree better with each other. However, the results for the Cosby and Slanger (2007) data are based on only 10 nights and the $v^{\prime}$-averaged October temperature was about $7 \mathrm{~K}$ higher. In comparison to our data, it has to be considered that their $T_{\text {rot }}$ are based on different line and band sets. Since the data do not show a robust $v^{\prime}$-dependent trend, an analysis of a larger sample is probably required to understand the significance and possible origin of the observed variations.

The vibrational populations of the four seasons relative to the annual mean are displayed in Fig. 13. As for $T_{\text {rot }}$, spring and autumn show the highest values. The lowest $\mathrm{OH}$ population is found in winter. Due to the clear separation of the four seasons, Fig. 13 can be best explained by a mixing of an annual and a semi-annual oscillation. This is consistent with the findings of Takahashi et al. (1995) for Cachoeira Paulista and Gelinas et al. (2008) for Alice Springs, although the positions of the extrema show some deviations. The amplitude of the seasonal variations in our data of about $20 \%$, which is a lower limit due to only four bins, is also in good agreement with the results of the listed studies. Figure 13 does not reveal any significant dependence of the population variations on $v^{\prime}$. Consequently, the $\mathrm{OH}$ vibrational level excitation and the subsequent relaxation processes appear to be relatively stable at Cerro Paranal in the course of a year, despite significant $\mathrm{OH}$ population changes.

\subsubsection{Correlations}

As final step of our analysis, we investigated how quantities derived from the individual observations of our sample were correlated. We considered the $\left\langle T_{\text {rot }}\left(v^{\prime}\right)\right\rangle$ already discussed, i.e. the mean rotational temperature of all $v^{\prime}$ from 2 to 9 (see Sect. 5.2.1), the slope $\Delta T_{\text {rot }} / \Delta v^{\prime}$ for all $v^{\prime}$, the population $n$ of $v^{\prime}=5$ relative to the sample mean $\langle n\rangle$, and the vibrational temperature $T_{\mathrm{vib}}$ derived from the levels $v^{\prime}=2$ to 9 . These parameters characterise the populations and temperatures for all accessible $v^{\prime}$. For $n /\langle n\rangle$, we only used the intermediate $v^{\prime}=5$ to avoid the bias of summed populations towards the dominating low $v^{\prime}$ (see Fig. 7). Table 2 lists the correlation coefficients $r$ for all combinations of the four quantities. The values were calculated for G98 as well as LG08 molecular data. The $r$ values agree quite well except for those combinations involving $\Delta T_{\text {rot }} / \Delta v^{\prime}$, where the LG08-related correlations are weaker. This is mainly due to the lower quality of the $T_{\text {rot }}$ especially for $v^{\prime}=2$ (see Sect. 4.2), which is crucial for calculating the slope. Student's $t$ tests for the deviation of the regression line slopes from 0 revealed significant $(\sigma>5)$ non-zero slopes for the investigated correlations with $|r|>0.3$.

Significant correlations exist for the majority of the combinations. An exception is $\left\langle T_{\text {rot }}\right\rangle$ vs. $\Delta T_{\text {rot }} / \Delta v^{\prime}$, where $r$ is close to 0 . Moreover, $\left\langle T_{\text {rot }}\right\rangle$ vs. $T_{\text {vib }}$ is not convincing. Consequently, the rotational temperature does not show a significant dependence on $v^{\prime}$-related quantities. However, there is a good correlation with the $\mathrm{OH}$ population measured by the intensity. This is well known (Takahashi et al., 1995; Gelinas et al., 2008; Reisin and Scheer, 2009; S. M. Smith, 2012), as it can be proved by observations of a single $\mathrm{OH}$ band. Temperature and intensity are affected by similar dynamical variations like the solar diurnal tide, the semi-annual oscillation, and gravity waves. Phase shifts between the changes in both quantities weaken the correlation. In particular, the nocturnal variations can show shifts of several hours (Gelinas et al., 2008). This is also supported by our results discussed in Sect. 5.2.1. On average, there was a $T_{\text {rot }}$ minimum in the first half of the night, whereas the intensity was decreasing during the entire night. An important contribution to the correlation is certainly the semi-annual oscillation with maxima in spring and autumn for both quantities (see Sect. 5.2.2). However, the annual oscillation was distinctly stronger for the intensity. These results are in good agreement with those obtained at a similar latitude as Cerro Paranal (Takahashi et al., 1995, 1998; Gelinas et al., 2008). 


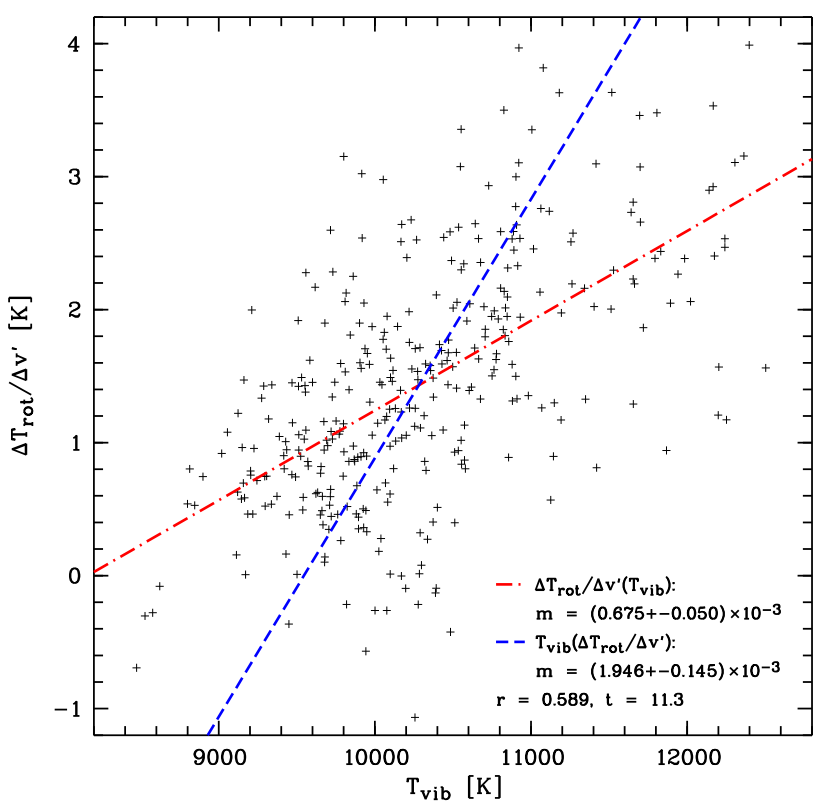

Figure 14. Change of $T_{\text {rot }}$ with respect to $v^{\prime} \Delta T_{\text {rot }} / \Delta v^{\prime}$ (cf. Fig. 10) vs. $T_{\text {vib }}$ for all $v^{\prime}$ for the individual X-shooter observations and G98 line parameters. The dash-dotted red and dashed blue lines show the results of an regression analysis (see legend).

Another good correlation was found for the change of $T_{\text {rot }}$

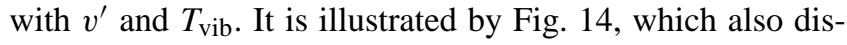
plays the results of an regression analysis. Although the scatter of the data points causes some uncertainties in the true slope of the regression line, the correlation is significant, as our $t$ test indicates. This result for the full sample confirms our findings for the example night shown in Fig. 8. It is also consistent with the result of Cosby and Slanger (2007) based on a single night. They interpreted the correlation as a consequence of the competition between the formation of excited $\mathrm{OH}$ in high $v$ with a hot rotational level distribution (Llewellyn and Long, 1978) and the rotational and vibrational relaxation processes by collisions. By radiative transitions, the rotational level can only change by one quantum at most. In the case of a relatively low frequency of collisions (especially for the high-altitude high $v$ ), this would lead to high $T_{\text {vib }}$ and $\Delta T_{\text {rot }} / \Delta v^{\prime}$. The latter would be due to a lower degree of thermalisation at higher $v$, where the influence of the nascent level distribution becomes larger. In other words, Cosby and Slanger (2007) assume non-LTE effects as the reason for the $T_{\text {rot }}$ differences. We have also suggested this for explaining the $T_{\text {rot }}$ differences for even and odd $v^{\prime}$ (Sects. 4.2 and 5.2.1). Moreover, we found a positive $\Delta T_{\text {rot }} / \Delta v^{\prime}$ for $95 \%$ of the sample spectra (see also Sect. 4.2), which contradicts the expected negative mean trend (Friedman and Chu, 2007; Clemesha et al., 2011) under the assumption of an increase in the $\mathrm{OH}$ emission altitude with $v^{\prime}$ (von Savigny et al., 2012). Nevertheless, true atmospheric temperature gradients could still play a role in the case of al- titude differences of several $\mathrm{km}$ for the $v^{\prime}$-related emission peaks, although it might be difficult to separate them from the non-LTE contributions.

$\Delta T_{\text {rot }} / \Delta v^{\prime}$ and $T_{\text {vib }}$ show an anticorrelation with the $v^{\prime}$ population or intensity. Consequently, the latter property is the only one which correlates with all the other quantities. This could be a hint for the $\mathrm{OH}$ intensity to be directly related to the source of the measured variations. As the intensity is related to the altitude of the $\mathrm{OH}$ peak emission (e.g. Liu and Shepherd, 2006), an intensity decrease could be explained by a rise of the emission layer (see also Sect. 5.1). Since the concentration of $\mathrm{O}_{2}$ decreases with altitude, collisional relaxation would become less efficient, which can then cause an increase of $\Delta T_{\text {rot }} / \Delta v^{\prime}$ and $T_{\text {vib }}$.

\section{Conclusions}

We used 343 high-quality spectra from medium-resolution echelle spectrograph X-shooter at Cerro Paranal in Chile in order to investigate $25 \mathrm{OH}$ bands between 0.58 and $2.24 \mu \mathrm{m}$. The simultaneous observations of these bands allowed us to characterise and compare upper level populations, rotational temperatures $T_{\text {rot }}$, and vibrational temperatures $T_{\text {vib }}$ and their change with time. In particular, we could study the reliability of $\mathrm{OH}$ rotational line populations as a proxy of the ambient temperature in the mesopause region.

The main results are as follows.

- Focusing on $P$-branch lines, we found a strong impact of the line set on the derived $T_{\text {rot }}$. Including lines of the fourth rotational level could already increase temperatures by up to $10 \%$. We also identified significantly lower rotational populations of the electronic substate $\mathrm{X}^{2} \Pi_{1 / 2}$ compared to $\mathrm{X}^{2} \Pi_{3 / 2}$ for a fixed rotational level energy. Hence, different mixing of $P_{1}$ and $P_{2}$ lines also affects $T_{\text {rot }}$. The rotational level populations did not show significant changes with time, which allowed for fixed temperature corrections for line set conversions.

- For a reference line set involving the first three $P$ branch lines of both electronic substates, we derived $T_{\text {rot }}$ for molecular line parameters of Goldman et al. (1998) and van der Loo and Groenenboom $(2007,2008)$. The choice of the line data caused differences by several K. However, these changes were similar for most bands. $\mathrm{OH}$ bands with the same upper vibrational level $v^{\prime}$ showed a $T_{\text {rot }}$ scatter of about $2 \mathrm{~K}$ for G98 data and about $4 \mathrm{~K}$ for LG08 data, which is most likely caused by measurement errors and uncertainties in the line parameters. The spectra revealed a robust pattern of $T_{\text {rot }}$ as a function of $v^{\prime}$ with higher $T_{\text {rot }}$ for even $v^{\prime}$ than for adjacent odd $v^{\prime}$. For even $v^{\prime}, T_{\text {rot }}$ strongly increased with $v^{\prime}$, which resulted in by far the highest temperatures for $v^{\prime}=8$.

- Vibrational level populations for a given $v^{\prime}$ depend on the $\mathrm{OH}$ band. The distribution is more extended for $\mathrm{G} 98$ 
than for LG08 data. The total populations for both line parameter sets also differ significantly. The vibrational level populations indicate a persistent relative overpopulation of the nascent levels 7 to 9 relative to the levels 3 to 6 if a Boltzmann distribution is assumed. The maximum overpopulation of about $40 \%$ was found for $v^{\prime}=8$. Hence, the slope of the population distribution measured by $T_{\mathrm{vib}}$ depends on the selected $v^{\prime}$ range.

- The average nocturnal variations are characterised by a $T_{\text {rot }}$ minimum before midnight and a steep intensity decrease at the beginning of the night. The variations are in good agreement with other observations at a similarly low latitude. The variability pattern changes with $v^{\prime}$ but is very similar for $\mathrm{OH}$ bands with the same $v^{\prime}$. The spread of $T_{\text {rot }}$ for the different $v^{\prime}$ shows a minimum at the beginning of the night and a rapid increase afterwards. The $T_{\text {rot }}$ difference between adjacent even and odd $v^{\prime}$ around $v^{\prime}=6$ also tends to rise. Moreover, $T_{\text {vib }}$ indicates an increase with time. For a night with good data coverage, we could also identify $v^{\prime}$-dependent phase shifts in $T_{\text {rot }}$ and intensity. There was a monotonic shift from $v^{\prime}=9$ to 2 .

- A rough investigation of seasonal variations revealed the predominance of a semi-annual oscillation, which is in agreement with other results at similar latitudes. The intensities also showed some contribution from an annual oscillation. Variations depending on $v^{\prime}$ are very weak. Only the change of $T_{\text {rot }}$ with $v^{\prime} \Delta T_{\text {rot }} / \Delta v^{\prime}$ could be smaller in spring than in the other seasons.

- The intensity indicates significant anticorrelations with $\Delta T_{\text {rot }} / \Delta v^{\prime}$ and $T_{\text {vib }}$, and a positive correlation with $T_{\text {rot }}$. For the latter, there is no convincing correlation with both $v^{\prime}$-dependent properties.

These results suggest that mesopause temperature measurements via $T_{\text {rot }}$ from $\mathrm{OH}$ bands suffer from systematic uncertainties of several K. First, the Einstein coefficients and level energies still show discrepancies. Improvements might be possible by comparing the results for $\mathrm{OH}$ bands with the same $v^{\prime}$. Second, the derived temperatures are significantly affected by non-LTE contributions which are particularly strong for high rotational levels and high even $v^{\prime}$, i.e. especially $v^{\prime}=8$. The $T_{\text {rot }}\left(v^{\prime}\right)$ pattern can be explained by remaining signatures of the nascent level population in an environment with inefficient thermalisation by collisions but a strong impact of radiative $v$ changes dominated by $\Delta v=2$ transitions. Temperatures depending on $v^{\prime}$ are possible due to different emission peak altitudes, which is confirmed by the observed $v^{\prime}$-dependent variability patterns. Higher altitudes with increasing $v^{\prime}$ can explain the almost always positive $\Delta T_{\text {rot }} / \Delta v^{\prime}$, since the concentration of the most important quencher for vibrational relaxation $\mathrm{O}_{2}$ decreases with altitude, i.e. the thermalisation becomes less efficient. The ambient temperature gradients for altitude differences of about
$0.5 \mathrm{~km}$ for $\Delta v^{\prime}=1$ (von Savigny et al., 2012), which should tend to be negative (Clemesha et al., 2011), appear to play a less important role. Consequently, mesopause temperatures and their gradients cannot reliably be derived from $\mathrm{OH} T_{\text {rot }}$ measurements if an accuracy better than several $K$ is desired. This supports results of Cosby and Slanger (2007) based on a smaller data set. Studies of the temperature variability are safer. However, even these measurements can be affected by non-LTE effects if the altitudes of the $v^{\prime}$-dependent emission layers change depending on the concentrations of the reactants and quenchers. Our data suggest a nocturnal $\mathrm{OH}$ layer rise in agreement with other results (e.g. Liu and Shepherd, 2006). The observed enhancement of non-LTE signatures in the $T_{\text {rot }}\left(v^{\prime}\right)$ distribution and higher $T_{\text {vib }}$ for decreasing intensity can be best explained by a decrease of the $\mathrm{O}_{2}$ concentration, i.e. higher altitudes. Therefore, all mesopause temperature estimates based on $\mathrm{OH} T_{\text {rot }}$ measurements should be taken with care, irrespective of the application. In this respect, it was a prudent decision to optimise the widely-used GRIPS instruments (e.g. Schmidt et al., 2013) for the OH(31 ) band and to focus on the first three $P_{1}$ lines, as $v^{\prime}=3$ appears to be less affected by non-LTE effects than the higher $v^{\prime}$.

\section{The Supplement related to this article is available online at doi:10.5194/acp-15-3647-2015-supplement.}

Acknowledgements. This project made use of the ESO Science Archive Facility. X-shooter spectra from different observing programmes of the period from October 2009 to March 2013 were used. We thank the two anonymous referees for their detailed and very helpful comments. This publication is supported by the Austrian Science Fund (FWF). S. Noll and S. Unterguggenberger receive funding from FWF project P26130. W. Kausch is funded by project IS538003 (Hochschulraumstrukturmittel) provided by the Austrian Ministry for Research (bmwfw), which also supports A. M. Jones via project BMWF-10.490/0008-II/3/2011.

Edited by: W. Ward 


\section{References}

Adler-Golden, S.: Kinetic parameters for $\mathrm{OH}$ nightglow modeling consistent with recent laboratory measurements, J. Geophys. Res., 102, 19969-19976, doi:10.1029/97JA01622, 1997.

Baker, D. J. and Stair Jr., A. T.: Rocket measurements of the altitude distributions of the hydroxyl airglow, Phys. Scripta, 37, 611-622, doi:10.1088/0031-8949/37/4/021, 1988.

Bates, D. R. and Nicolet, M.: The photochemistry of atmospheric water vapor, J. Geophys. Res., 55, 301-327, doi:10.1029/JZ055i003p00301, 1950.

Beig, G., Keckhut, P., Lowe, R. P., Roble, R. G., Mlynczak, M. G., Scheer, J., Fomichev, V. I., Offermann, D., French, W. J. R., Shepherd, M. G., Semenov, A. I., Remsberg, E. E., She, C. Y., Lübken, F. J., Bremer, J., Clemesha, B. R., Stegman, J., Sigernes, F., and Fadnavis, S.: Review of mesospheric temperature trends, Rev. Geophys., 41, RG1015, doi:10.1029/2002RG000121, 2003.

Beig, G., Scheer, J., Mlynczak, M. G., and Keckhut, P.: Overview of the temperature response in the mesosphere and lower thermosphere to solar activity, Rev. Geophys., 46, RG3002, doi:10.1029/2007RG000236, 2008.

Burrage, M. D., Hagan, M. E., Skinner, W. R., Wu, D. L., and Hays, P. B.: Long-term variability in the solar diurnal tide observed by HRDI and simulated by the GSWM, Geophys. Res. Lett., 22, 2641-2644, doi:10.1029/95GL02635, 1995.

Chamberlain, J. W.: Physics of the Aurora and Airglow, Academic Press, New York, USA, 1961.

Charters, P. E., MacDonald, R. G., and Polanyi, J. C.: Formation of vibrationally excited $\mathrm{OH}$ by the reaction $\mathrm{H}+\mathrm{O}_{3}$, Appl. Optics, 10, 1747-1754, doi:10.1364/AO.10.001747, 1971.

Clemesha, B., Simonich, D., and Batista, P.: Sodium lidar measurements of mesopause region temperatures at $23^{\circ} \mathrm{S}$, Adv. Space Res., 47, 1165-1171, doi:10.1016/j.asr.2010.11.030, 2011.

Clough, S. A., Shephard, M. W., Mlawer, E. J., Delamere, J. S., Iacono, M. J., Cady-Pereira, K., Boukabara, S., and Brown, P. D.: Atmospheric radiative transfer modeling: a summary of the AER codes, J. Quant. Spectrosc. Ra., 91, 233-244, doi:10.1016/j.jqsrt.2004.05.058, 2005.

Cosby, P. C. and Slanger, T. G.: OH spectroscopy and chemistry investigated with astronomical sky spectra, Can. J. Phys., 85, 7799, 2007.

Cosby, P. C., Sharpee, B. D., Slanger, T. G., Huestis, D. L., and Hanuschik, R. W.: High-resolution terrestrial nightglow emission line atlas from UVES/VLT: Positions, intensities, and identifications for 2808 lines at $314-1043 \mathrm{~nm}$, J. Geophys. Res., 111, A12307, doi:10.1029/2006JA012023, 2006.

Dekker, H., D'Odorico, S., Kaufer, A., Delabre, B., and Kotzlowski, H.: Design, construction, and performance of UVES, the echelle spectrograph for the UT2 Kueyen Telescope at the ESO Paranal Observatory, SPIE Proc. Ser., 4008, 534-545, 2000.

Friedman, J. S. and Chu, X.: Nocturnal temperature structure in the mesopause region over the Arecibo Observatory $\left(18.35^{\circ} \mathrm{N}\right.$, $\left.66.75^{\circ} \mathrm{W}\right)$ : Seasonal variations, J. Geophys. Res., 112, D14107, doi:10.1029/2006JD008220, 2007.

Gelinas, L. J., Hecht, J. H., Walterscheid, R. L., Roble, R. G., and Woithe, J. M.: A seasonal study of mesospheric temperatures and emission intensities at Adelaide and Alice Springs, J. Geophys. Res., 113, A01304, doi:10.1029/2007JA012587, 2008.
Goldman, A., Schoenfeld, W. G., Goorvitch, D., Chackerian Jr., C., Dothe, H., Mélen, F., Abrams, M. C., and Selby, J. E. A.: Updated line parameters for $\mathrm{OH} \mathrm{X}^{2} \mathrm{II}-\mathrm{X}^{2} \mathrm{II}\left(v^{\prime}, v^{\prime \prime}\right)$ transitions, J. Quant. Spectrosc. Ra., 59, 453-469, doi:10.1016/S00224073(97)00112-X, 1998.

Hanuschik, R. W.: A flux-calibrated, high-resolution atlas of optical sky emission from UVES, Astron. Astrophys., 407, 1157-1164, doi:10.1051/0004-6361:20030885, 2003.

Jones, A., Noll, S., Kausch, W., Szyszka, C., and Kimeswenger, S.: An advanced scattered moonlight model for Cerro Paranal, Astron. Astrophys., 560, A91, doi:10.1051/0004-6361/201322433, 2013.

Jones, A., Noll, S., Kausch, W., Szyszka, C., and Kimeswenger, S.: An advanced scattered moonlight model, The Messenger, 156, 31-34, 2014.

Kausch, W., Noll, S., Smette, A., Kimeswenger, S., Barden, M., Szyszka, C., Jones, A. M., Sana, H., Horst, H., and Kerber, F.: Molecfit: a general tool for telluric absorption correction, II. Quantitative evaluation on ESO-VLT/X-shooter spectra, Astron. Astrophys., 576, A78, doi:10.1051/0004-6361/201423909, 2015.

Khomich, V. Y., Semenov, A. I., and Shefov, N. N.: Airglow as an Indicator of Upper Atmospheric Structure and Dynamics, Springer, Berlin, Germany, 2008.

Kowalewski, S., von Savigny, C., Palm, M., McDade, I. C., and Notholt, J.: On the impact of the temporal variability of the collisional quenching process on the mesospheric $\mathrm{OH}$ emission layer: a study based on SD-WACCM4 and SABER, Atmos. Chem. Phys., 14, 10193-10210, doi:10.5194/acp-14-10193-2014, 2014.

Krassovsky, V. I., Potapov, B. P., Semenov, A. I., Sobolev, V. G., Shagaev, M. V., and Shefov, N. N.: On the equilibrium nature of the rotational temperature of hydroxyl airglow, Planet. Space Sci., 25, 596-597, doi:10.1016/0032-0633(77)90067-8, 1977.

Liu, G. and Shepherd, G. G.: An empirical model for the altitude of the $\mathrm{OH}$ nightglow emission, Geophys. Res. Lett., 33, L09805, doi:10.1029/2005GL025297, 2006.

Llewellyn, E. J. and Long, B. H.: The OH Meinel bands in the airglow - the radiative lifetime, Can. J. Phys., 56, 581-586, doi:10.1139/p78-076, 1978.

Lopez-Moreno, J. J., Rodrigo, R., Moreno, F., Lopez-Puertas, M., and Molina, A.: Altitude distribution of vibrationally excited states of atmospheric hydroxyl at levels $v=2$ to $v=7$, Planet. Space Sci., 35, 1029-1038, doi:10.1016/0032-0633(87)90007-9, 1987.

Marsh, D. R., Smith, A. K., Mlynczak, M. G., and Russell, J. M.: SABER observations of the $\mathrm{OH}$ Meinel airglow variability near the mesopause, J. Geophys. Res., 111, A10S05, doi:10.1029/2005JA011451, 2006.

McDade, I. C.: The altitude dependence of the $\mathrm{OH}\left(\mathrm{X}^{2} \Pi\right)$ vibrational distribution in the nightglow - some model expectations, Planet. Space Sci., 39, 1049-1057, doi:10.1016/00320633(91)90112-N, 1991.

McDade, I. C. and Llewellyn, E. J.: Kinetic parameters related to sources and sinks of vibrationally excited $\mathrm{OH}$ in the nightglow, $\mathrm{J}$ Geophys. Res., 92, 7643-7650, doi:10.1029/JA092iA07p07643, 1987.

Meinel, A. B.: OH emission bands in the spectrum of the night sky. I, Astrophys. J., 111, 555-564, doi:10.1086/145296, 1950a. 
Meinel, A. B.: $\mathrm{OH}$ emission bands in the spectrum of the night sky. II, Astrophys. J., 112, 120-130, doi:10.1086/145321, 1950b.

Melo, S. M. L., Lowe, R. P. and Takahashi, H.: The nocturnal behavior of the hydroxyl airglow at the equatorial and low latitudes as observed by WINDII: Comparison with groundbased measurements, J. Geophys. Res., 104, 24657-24666, doi:10.1029/1999JA900291, 1999.

Meriwether, J. W. and Gardner, C. S.: A review of the mesosphere inversion layer phenomenon, J. Geophys. Res., 105, 1240512416, doi:10.1029/2000JD900163, 2000.

Mies, F. H.: Calculated vibrational transition probabilities of $\mathrm{OH}\left(\mathrm{X}^{2} \Pi\right)$, J. Mol. Spectrosc., 53, 150, doi:10.1016/00222852(74)90125-8, 1974

Modigliani, A., Goldoni, P., Royer, F., Haigron, R., Guglielmi, L., François, P., Horrobin, M., Bristow, P., Vernet, J., Moehler, S., Kerber, F., Ballester, P., Mason, E., and Christensen, L.: The X-shooter pipeline, SPIE Proc. Ser., 7737, 773728, doi:10.1117/12.857211, 2010.

Moehler, S., Modigliani, A., Freudling, W., Giammichele, N., Gianninas, A., Gonneau, A., Kausch, W., Lançon, A., Noll, S., Rauch, T., and Vinther, J.: Flux calibration of mediumresolution spectra from $300 \mathrm{~nm}$ to $2500 \mathrm{~nm}$ : Model reference spectra and telluric correction, Astron. Astrophys., 568, A9, doi:10.1051/0004-6361/201423790, 2014.

Noll, S., Kausch, W., Barden, M., Jones, A. M., Szyszka, C., Kimeswenger, S., and Vinther, J.: An atmospheric radiation model for Cerro Paranal. I. The optical spectral range, Astron. Astrophys., 543, A92, doi:10.1051/0004-6361/201219040, 2012

Noll, S., Kausch, W., Kimeswenger, S., Barden, M., Jones, A. M., Modigliani, A., Szyszka, C., and Taylor, J.: Skycorr: a general tool for spectroscopic sky subtraction, Astron. Astrophys., 567, A25, doi:10.1051/0004-6361/201423908, 2014.

Ohoyama, H., Kasai, T., Yoshimura, Y., Kimura, H., and Kuwata, K.: Initial distribution of vibration of the $\mathrm{OH}$ radicals produced in the $\mathrm{H}+\mathrm{O}_{3} \rightarrow \mathrm{OH}\left(\mathrm{X}^{2} \Pi_{1 / 2,3 / 2}\right)+\mathrm{O}_{2}$ reaction. Chemiluminescence by a crossed beam technique, Chem. Phys., 118, 263-266, doi:10.1016/0009-2614(85)85312-4, 1985.

Osterbrock, D. E., Fulbright, J. P., Martel, A. R., Keane, M. J., Trager, S. C., and Basri, G.: Night-Sky High-Resolution Spectral Atlas of $\mathrm{OH}$ and $\mathrm{O}_{2}$ Emission Lines for Echelle Spectrograph Wavelength Calibration, Publ. Astron. Soc. Pac., 108, 277-308, 1996.

Patat, F.: The dancing sky: 6 years of night-sky observations at Cerro Paranal, Astron. Astrophys., 481, 575-591, doi:10.1051/0004-6361:20079279, 2008.

Patat, F., Moehler, S., O'Brien, K., Pompei, E., Bensby, T., Carraro, G., de Ugarte Postigo, A., Fox, A., Gavignaud, I., James, G., Korhonen, H., Ledoux, C., Randall, S., Sana, H., Smoker, J., Stefl, S., and Szeifert, T.: Optical atmospheric extinction over Cerro Paranal, Astron. Astrophys., 527, A91, doi:10.1051/00046361/201015537, 2011.

Pendleton Jr., W., Espy, P., Baker, D., Steed, A., and Fetrow, M.: Observation of $\mathrm{OH}$ Meinel $(7,4) \mathrm{P}(\mathrm{N}$-double-prime $=13)$ transitions in the night airglow, J. Geophys. Res., 94, 505-510, doi:10.1029/JA094iA01p00505, 1989.

Pendleton Jr., W. R., Espy, P. J., and Hammond, M. R.: Evidence for non-local-thermodynamic-equilibrium rotation in the $\mathrm{OH}$ nightglow, J. Geophys. Res., 98, 11567-11579, doi:10.1029/93JA00740, 1993.

Perminov, V. I. and Semenov, A. I.: Nonequilibrium of the rotational temperature of $\mathrm{OH}$ bands with high vibrational excitation, Geomagn. Aeron., 32, 175-178, 1992.

Perminov, V. I., Semenov, A. I., and Shefov, N. N.: On rotational temperature of the hydroxyl emission, Geomagn. Aeron., 47, 756-763, doi:10.1134/S0016793207060084, 2007.

Reisin, E. R. and Scheer, J.: Evidence of change after 2001 in the seasonal behaviour of the mesopause region from airglow data at El Leoncito, Adv. Space Res., 44, 401-412, doi:10.1016/j.asr.2009.04.007, 2009.

Rothman, L. S., Gordon, I. E., Barbe, A., Chris Benner, D., Bernath, P. F., Birk, M., Boudon, V., Brown, L. R., Campargue, A., Champion, J.-P., Chance, K., Coudert, L. H., Dana, V., Devi, V. M., Fally, S., Flaud, J.-M., Gamache, R. R., Goldman, A., Jacquemart, D., Kleiner, I., Lacome, N., Lafferty, W. J., Mandin, J.-Y., Massie, S. T., Mikhailenko, S. N., Miller, C. E., Moazzen-Ahmadi, N., Naumenko, O. V., Nikitin, A. V., Orphal, J., Perevalov, V. I., Perrin, A., Predoi-Cross, A., Rinsland, C. P., Rotger, M., Šimečková, M., Smith, M. A. H. Sung, K., Tashkun, S. A., Tennyson, J., Toth, R. A., Vandaele, A. C., and Vander Auwera, J.: The HITRAN 2008 molecular spectroscopic database, J. Quant. Spectrosc. Ra., 110, 533572, doi:10.1016/j.jqsrt.2009.02.013, 2009.

Rothman, L. S., Gordon, I. E., Babikov, Y., Barbe, A., Chris Benner, D., Bernath, P. F., Birk, M., Bizzocchi, L., Boudon, V., Brown, L. R., Campargue, A., Chance, K., Cohen, E. A., Coudert, L. H., Devi, V. M., Drouin, B. J., Fayt, A., Flaud, J.M., Gamache, R. R., Harrison, J. J., Hartmann, J.-M., Hill, C., Hodges, J. T., Jacquemart, D., Jolly, A., Lamouroux, J., Le Roy, R. J., Li, G., Long, D. A., Lyulin, O. M., Mackie, C. J., Massie, S. T., Mikhailenko, S., Müller, H. S. P., Naumenko, O. V., Nikitin, A. V., Orphal, J., Perevalov, V., Perrin, A., Polovtseva, E. R., Richard, C., Smith, M. A. H., Starikova, E., Sung, K., Tashkun, S., Tennyson, J., Toon, G. C., Tyuterev, V. G., and Wagner, G.: The HITRAN2012 molecular spectroscopic database, J. Quant. Spectrosc. Ra., 130, 4-50, doi:10.1016/j.jqsrt.2013.07.002, 2013.

Rousselot, P., Lidman, C., Cuby, J.-G., Moreels, G., and Monnet, G.: Night-sky spectral atlas of $\mathrm{OH}$ emission lines in the nearinfrared, Astron. Astrophys., 354, 1134-1150, 2000.

Rozenberg, G. V.: Twilight: a Study in Atmospheric Optics, Plenum Press, New York, USA, 1966

Schmidt, C., Höppner, K., and Bittner, M.: A ground-based spectrometer equipped with an InGaAs array for routine observations of $\mathrm{OH}(3-1)$ rotational temperatures in the mesopause region, J. Atmos. Sol.-Terr. Phy., 102, 125-139, doi:10.1016/j.jastp.2013.05.001, 2013.

Sheinis, A. I., Bolte, M., Epps, H. W., Kibrick, R. I., Miller, J. S., Radovan, M. V., Bigelow, B. C., and Sutin, B. M.: ESI, a New Keck Observatory Echellette Spectrograph and Imager, Publ. Astron. Soc. Pac., 114, 851-865, doi:10.1086/341706, 2002.

Smette, A., Sana, H., Noll, S., Horst, H., Kausch, W., Kimeswenger, S., Barden, M., Szyszka, C., Jones, A. M., Gallenne, A., Vinther, J., Ballester, P., and Taylor, J.: Molecfit: a general tool for telluric absorption correction. I. Method and application to ESO instruments, Astron. Astrophys., 576, A77, doi:10.1051/0004-6361/201423932, 2015. 
Smith, A. K., Marsh, D. R., Mlynczak, M. G., and Mast, J. C.: Temporal variations of atomic oxygen in the upper mesosphere from SABER, J. Geophys. Res., 115, D18309, doi:10.1029/2009JD013434, 2010.

Smith, A. K.: Global Dynamics of the MLT, Surv. Geophys., 33, 1177-1230, doi:10.1007/s10712-012-9196-9, 2012.

Smith, S. M.: Seasonal variations in the correlation of mesospheric $\mathrm{OH}$ temperature and radiance at midlatitudes, J. Geophys. Res., 117, A10308, doi:10.1029/2012JA017884, 2012.

Takahashi, H. and Batista, P. P.: Simultaneous measurements of $\mathrm{OH}(9-4),(8-3),(7-2),(6-2)$ and (5-1) bands in the airglow, J. Geophys. Res., 86, 5632-5642, doi:10.1029/JA086iA07p05632, 1981.

Takahashi, H., Clemesha, B. R., and Batista, P. P.: Predominant semi-annual oscillation of the upper mesospheric airglow intensities and temperatures in the equatorial region, J. Atmos. Terr. Phys., 57, 407-414, 1995.

Takahashi, H., Gobbi, D., Batista, P. P., Melo, S. M. L., Teixeira, N. R., and Buriti, R. A.: Dynamical influence on the equatorial airglow observed from the south american sector, Adv. Space Res., 2, 817-825, doi:10.1016/S0273-1177(97)00680-7, 1998.

Taylor, M. J., Pendleton Jr., W. R., Clark., S., Takahashi, H., Gobbi, D., and Goldberg, R. A.: Image measurements of shortperiod gravity waves at equatorial latitudes, J. Geophys. Res., 102, 26283-26299, doi:10.1029/96JD03515, 1997.

Turnbull, D. N. and Lowe, R. P.: New hydroxyl transition probabilities and their importance in airglow studies, Planet. Space Sci., 37, 723-738, doi:10.1016/0032-0633(89)90042-1, 1989.

van der Loo, M. P. J. and Groenenboom, G. C.: Theoretical transition probabilities for the $\mathrm{OH}$ Meinel system, J. Chem. Phys., 126, 114314, doi:10.1063/1.2646859, 2007.

van der Loo, M. P. J. and Groenenboom, G. C.: Erratum: "Theoretical transition probabilities for the $\mathrm{OH}$ Meinel system" [J. Chem. Phys. 126, 114314], J. Chem. Phys., 128, 159902, doi:10.1063/1.2899016, 2008.

van Rhijn, P. J.: On the brightness of the sky at night and the total amount of starlight, Publ. Astr. Lab., Groningen, 31, 1-83, 1921.

Varandas, A. J. C.: Reactive and non-reactive vibrational quenching in $\mathrm{O}+\mathrm{OH}$ collisions, Chem. Phys. Lett., 396, 182-190, doi:10.1016/j.cplett.2004.08.023, 2004.
Vernet, J., Dekker, D’Odorico, S., Kaper, L., Kjaergaard, P., Hammer, F., Randich, S., Zerbi, F., Groot, P. M., Hjorth, J., Guinouard, I., Navarro, R., Adolfse, T., Albers, P. W., Amans, J.P., Andersen, J. J., Andersen, M. I., Binetruy, P., Bristow, P., Castillo, R., Chemla, F., Christensen, L., Conconi, P., Conzelmann, R., Dam, J., De Caprio, V., De Ugarte Postigo, A., Delabre, B., Di Marcantonio, P., Downing, M., Elswijk, E., Finger, G., Fischer, G., Flores, H., François, P., Goldoni, P., Guglielmi, L., Haigron, R., Hanenburg, H., Hendriks, I., Horrobin, M., Horville, D., Jessen, N. C., Kerber, F., Kern, L., Kiekebusch, M., Kleszcz, P., Klougart, J., Kragt, J., Larsen, H. H., Lizon, J.-L., Lucuix, C., Mainieri, V., Manuputy, R., Martayan, C., Mason, E., Mazzoleni, R., Michaelsen, N., Modigliani, A., Moehler, S., Møller, P., Norup Sørensen, A., Nørregaard, P., Péroux, C., Patat, F., Pena, E., Pragt, J., Reinero, C., Rigal, F., Riva, M., Roelfsema, R., Royer, F., Sacco, G., Santin, P., Schoenmaker, T., Spano, P., Sweers, E., Ter Horst, R., Tintori, M., Tromp, N., van Dael, P., van der Vliet, H., Venema, L., Vidali, M., Vinther, J., Vola, P., Winters, R., Wistisen, D., Wulterkens, G., and Zacchei, A.: X-shooter, the new wide band intermediate resolution spectrograph at the ESO Very Large Telescope, Astron. Astrophys., 536, A105, doi:10.1051/00046361/201117752, 2011.

Vincent, R. A., Tsuda, T., and Kato, S.: A comparative study of mesospheric solar tides observed at Adelaide and Kyoto, J. Geophys. Res., 93, 699-708, doi:10.1029/JD093iD01p00699, 1988.

von Savigny, C. and Lednyts'kyy, O.: On the relationship between atomic oxygen and vertical shifts between $\mathrm{OH}$ Meinel bands originating from different vibrational levels, Geophys. Res. Lett., 40, 5821-5825, doi:10.1002/2013GL058017, 2013

von Savigny, C., McDade, I. C., Eichmann, K.-U., and Burrows, J. P.: On the dependence of the $\mathrm{OH}^{*}$ Meinel emission altitude on vibrational level: SCIAMACHY observations and model simulations, Atmos. Chem. Phys., 12, 8813-8828, doi:10.5194/acp-128813-2012, 2012.

Xu, J., Gao, H., Smith, A. K., and Zhu, Y.: Using TIMED/SABER nightglow observations to investigate hydroxyl emission mechanisms in the mesopause region, J. Geophys. Res., 117, D02301, doi:10.1029/2011JD016342, 2012.

Yee, J.-H., Crowley, G., Roble, R. G., Skinner, W. R., Burrage, M. D., and Hays, P. B.: Global simulations and observations of $\mathrm{O}\left({ }^{1} \mathrm{~S}\right), \mathrm{O}_{2}\left({ }^{1} \Sigma\right)$ and $\mathrm{OH}$ mesospheric nightglow emissions, J. Geophys. Res., 102, 19949-19968, doi:10.1029/96JA01833, 1997. 